\title{
PERIODIC TRAVELING WAVES OF THE REGULARIZED SHORT PULSE AND OSTROVSKY EQUATIONS: EXISTENCE AND STABILITY*
}

\author{
SEVDZHAN HAKKAEV ${ }^{\dagger}$, MILENA STANISLAVOVA ${ }^{\ddagger}$ AND ATANAS STEFANOV $\ddagger$
}

\begin{abstract}
We construct various periodic traveling wave solutions of the Ostrovsky/HunterSaxton/short pulse equation and its KdV regularized version. For the regularized short pulse model with small Coriolis parameter, we describe a family of periodic traveling waves which are a perturbation of appropriate KdV solitary waves. We show that these waves are spectrally stable. For the short pulse model, we construct a family of traveling peakons with corner crests. We show that the peakons are spectrally stable as well.
\end{abstract}

Key words. spectral stability, traveling waves, peakons, short pulse equation, regularized short pulse equation

AMS subject classifications. 35B35, 35B40, 35G30

DOI. $10.1137 / 15 \mathrm{M} 1037901$

1. Introduction. It is well known that the (generalized) Korteweg-de Vries $(\mathrm{KdV})$ equation

$$
u_{t}+\beta u_{x x x}+(f(u))_{x}=0
$$

can be used in modeling and understanding the dynamics of large scales in the atmosphere and in the oceans. There is a substantial amount of research into various aspects of this model - well-posedness, propagation of singularities, asymptotic behavior near solitary waves, etc. We will not even attempt to review these here, as this is outside the scope of this paper. Instead, we discuss a related model, which takes into account the effect of a rotation force, if such is applied to the fluid. To be sure, the effect of the rotation of the Earth (which induces the so-called Coriolis force) is often a negligible factor. As such, it is not taken into account in the derivation of various water wave models, such as (1). However, adding this feature in the model results in the following dispersive model:

$$
\left(u_{t}+\beta u_{x x x}+(f(u))_{x}\right)_{x}+\epsilon u=0,-T \leq x \leq T .
$$

Here, $T$ could be finite or infinite, depending on the particular setup that one is interested in. We adopt the notation $\epsilon$ in order to emphasize the fact that usually $0<|\epsilon|<<1$ is a small parameter and also, at least mathematically, we can allow $\epsilon$ to

${ }^{*}$ Received by the editors September 2, 2015; accepted for publication (in revised form) August 18, 2016; published electronically February 28, 2017.

http://www.siam.org/journals/sima/49-1/M103790.html

Funding: The research of the second author was supported in part by NSF-DMS, Applied Mathematics program, under grants 1211315 and 1516245. The research of the third author was supported by NSF-DMS, Applied Mathematics program, under grant 1313107.

${ }^{\dagger}$ Faculty of Arts and Sciences, Department of Mathematics and Computer Science, Istanbul Aydin University, Istanbul, 81120, Turkey, and Faculty of Mathematics and Informatics, Shumen University, Shumen, Bulgaria (shakkaev@fmi.shu-bg.net).

${ }_{\ddagger}^{\ddagger}$ Department of Mathematics, University of Kansas, Lawrence, KS 66045-7523 (stanis@ku.edu, stefanov@ku.edu). 
be both positive and negative. ${ }^{1}$ This is a model which has gained popularity lately. ${ }^{2}$ We refer to it as the regularized short pulse equation (RSPE).

We should also mention that traditionally in the physics literature these models have been considered in finite spatially symmetric intervals, with appropriate boundary conditions. In addition, since (2) clearly forces the solution to be an exact derivative, one faces some mathematical difficulties associated with it, if one considers the problem on the whole line. More precisely, in the whole line context, even though one may still conclude from (5) that $\int_{-\infty}^{\infty} u(t, x) d x=0$, this is not enough to properly define $\partial_{x}^{-1} u$. Indeed, writing $\partial_{x}^{-1} u$ requires that $u \in \dot{H}^{-1}\left(\mathbf{R}^{1}\right)$, which is not a property easily enforced on the solutions of (2) for $t>0$. Thus, the reformulation of (2) in the form

$$
u_{t}+\beta u_{x x x}+(f(u))_{x}+\epsilon \partial_{x}^{-1} u=0
$$

which is somewhat desirable creates some technical difficulties in the well-posedness as well as the stability considerations in the whole line case. We will, however, consider the problem (3) in the periodic context, for which the operator $\partial_{x}^{-1}$ makes perfect sense on mean value zero functions.

Regardless of the issues associated with the theory in the whole line, there are several results regarding existence (and in some cases stability) of traveling waves. In $[18,19]$ (under some assumptions on the coefficient $\beta$, the function $f$, and $\epsilon$ ), Levandosky and Liu have succeeded in constructing traveling wave solutions of (2) on the whole line by employing variational methods. They have also studied the stability of such solutions by relying on the Grillakis-Shatah-Strauss theory, although their results do not give a full stability picture of all solitary waves constructed in their paper. Further work on the stability of these traveling waves was done by Liu, [20] and Liu and Ohta, [21].

In [7], the authors have considered the same problem for small values of $\epsilon$. They have constructed solutions of (5) via singular perturbation theory. In [8], in collaboration with Sandstede, they have extended their previous results to include the existence of multipulse solutions. To the best of our knowledge, the problem for the stability of both types of waves remains open.

An interesting special model occurs when the KdV regularization is absent, ${ }^{3}$ in other words, $\beta=0$. This is referred to in the literature, depending on the form of the nonlinearity $f$, as the reduced Ostrovsky/Ostrovsky-Hunter/short pulse model. Namely, we scale for convenience $\epsilon=1$, which leads us to

$$
\left(u_{t}+(f(u))_{x}\right)_{x}=u \text {. }
$$

Such a model represents an independent interest, not necessarily related to the (generalized) KdV equation. In fact, (4), with various forms of nonlinearity, has a rich history. The first model of this family was introduced by Ostrovsky [25] in the late 70s. In the early 90s, Vakhnenko [32] came up with an alternative derivation, while Hunter [13] proposed some numerical simulations. The well-posedness questions were investigated by Boyd [2], Schaefer and Wayne [29], and Stefanov, Shen,

\footnotetext{
${ }^{1}$ Although in physically relevant models, $\epsilon$ is a small negative number.

${ }^{2}$ One particularly interesting aspect of it is the connection to the two-dimensional KadomtsevPetviashvili (KP) equations - indeed, (2) provides special one-dimensional solutions of KP, which may be used as a reference in the study of the dynamics of KP.

${ }^{3}$ One should note that the presence of $\beta \neq 0$ provides a regularizing effect on the evolution [10].
} 
and Kevrekidis [30]. Liu, Pelinovsky, and Sakovich [22, 23] have studied wave breaking, which was later supplemented by the global regularity results of Grimshaw and Pelinovsky, [11]. There are numerous works on explicit traveling wave solutions of these models, [10, 24, 27, 28, 31, 33, 34]. One should note that some of these solutions are not classical solutions but rather are multivalued ones [28]. Several authors have also explored the integrability of the Ostrovsky equation [28, 34]; in particular they have managed to construct the traveling waves by means of the inverse scattering transform. Finally, there are several attempts at stability, like [26], [27]. These mostly consist of either direct numerical simulations of the full equation or numerical spectral computations, performed on the linearized operator.

Since we will be interested in traveling wave solutions of (2) and their stability properties, we should give a more precise definition and record the corresponding elliptic equations. More specifically, we consider solutions of (2) in the form $\varphi(x-c t)$. They satisfy

$$
-c \varphi^{\prime \prime}+\beta \varphi^{\prime \prime \prime \prime}+(f(\varphi))^{\prime \prime}+\epsilon \varphi=0,-T \leq x \leq T .
$$

In the periodic context and if one assume that $\varphi$ is sufficiently smooth, this formulation immediately implies that $\int_{-T}^{T} \varphi(x) d x=0$.

1.1. Some notation. We will often work in the subspace $L_{0}^{2}=\left\{f \in L^{2}[-T, T]\right.$ : $\left.\int_{-T}^{T} f(x) d x=0\right\}$, which can be realized as the space of all Fourier series with the restriction $a_{0}=0$. Namely, $f \in L_{0}^{2}$ if and only if $f(x)=\frac{1}{\sqrt{2 T}} \sum_{k=-\infty, k \neq 0}^{\infty} a_{k} e^{\pi i k x / T}$, with a norm given by

$$
\|f\|_{L_{0}^{2}}=\left(\sum_{k=-\infty, k \neq 0}^{\infty}\left|a_{k}\right|^{2}\right)^{1 / 2},
$$

where $a_{k}=\frac{1}{\sqrt{2 T}} \int_{-T}^{T} f(x) e^{-\pi i k x / T} d x$. The Sobolev spaces with mean zero functions are defined as follows: $H_{0}^{s}[-T, T]=L_{0}^{2} \cap H^{s}[-T, T]$. In addition, one can consider the action of the operator $\partial_{x}^{-1}$ on $L_{0}^{2}$ and the corresponding Sobolev spaces $H_{0}^{s}$, defined in a standard way by the formula

$$
\partial_{x}^{-1} f=T \sum_{k=-\infty, k \neq 0}^{\infty} \frac{a_{k}}{\pi i k} e^{\pi i k x / T} .
$$

More generally, we introduce the following operators, which will be useful in what follows. On the Sobolev space with zero mean functions, $H_{0}^{s}$ (where $s$ is a real parameter), let

$$
\left|\partial_{x}\right|^{s} f(x):=T^{-s} \sum_{k=-\infty, k \neq 0}^{\infty} \pi^{s}|k|^{s} a_{k} e^{\pi i k x / T} .
$$

In addition, introduce the Hilbert transform operator $H: H \partial_{x}=\left|\partial_{x}\right|$ or $\partial_{x}=-H\left|\partial_{x}\right|$. In other words,

$$
H f(x)=i \sum_{k=-\infty, k \neq 0}^{\infty} \operatorname{sgn}(k) a_{k} e^{\pi i k x / T} .
$$

It is easy to see that $H$ is a skew-symmetric operator, that is, $H^{*}=-H$. In addition, $H$ maps real-valued functions into real-valued functions. 
1.2. Organization of the paper and a synopsis of the main results. The main goal of this work is to provide some new periodic traveling wave solutions of the short pulse equation (4) and its regularized version, (2). For the RSPE (2), we can construct a family of waves, for all small $\epsilon$, whenever the underlying $\mathrm{KdV}$ equation (1) has such waves and they satisfy appropriate nondegeneracy requirements. This is done in section 2 below via the Lyapunov-Schmidt reduction argument. We apply these results, for example, ${ }^{4}$ to the cnoidal solutions of KdV constructed in section 2.1 . In section 3, we establish the spectral stability of the solutions constructed perturbatively in section 2. It is perhaps interesting to note that the solutions constructed in this way, as perturbation of the corresponding ( $\mathrm{gKdV}$ ) models, have the same stability properties as the original solitons, modulo some mild technical assumptions. Our approach relies on the Hamilton-Krein index theory, developed by Kapitula, Kevrekidis, and Sandstede in the works $[15,16]$; see also the related work of Chugunova and Pelinovsky [6].

In section 4 , we consider the Ostrovsky equation (4), in the case $f(u)=-u^{2}$. We construct peakon type solutions. These are continuous functions in an interval $[-T, T]$, but their derivatives develop a jump discontinuity at the points $\pm T$. That is, they have a corner crest. In section 5 , we show that these periodic traveling peakons are spectrally stable.

2. Construction of the periodic traveling waves for the RSPE with small Coriolis parameter. In this section, we first state and prove a general theorem for the existence of periodic traveling waves for RSPE, for small $\epsilon:|\epsilon|<<1$. After that, we give concrete and explicit forms of the traveling wave solutions of the $\mathrm{KdV}$ equation, which satisfy the assumption in Theorem 1 below.

Before we formulate the main result, let us rewrite (5) in a more suitable form for our analysis. For $\epsilon=0,(5)$ reduces to $-\partial_{x}^{2}\left[-\beta \varphi^{\prime \prime}+c \varphi-f(\varphi)\right]=0$. After two integrations in the $x$ variable, this reduces ${ }^{5}$ to

$$
-\beta \varphi^{\prime \prime}+c \varphi-f(\varphi)=h, \quad-T \leq x \leq T .
$$

For the general case, under the condition $\int_{-T}^{T} \varphi(x) d x=0$, we are looking for a solution in the form

$$
-\beta \varphi_{\epsilon}^{\prime \prime}+c \varphi_{\epsilon}-f\left(\varphi_{\epsilon}\right)-\epsilon \partial_{x}^{-2} \varphi_{\epsilon}=h+\alpha(\epsilon), \varphi_{\epsilon} \in L_{0}^{2}[-T, T]
$$

for some $\alpha=\alpha(\epsilon)$. Indeed, any solution of (9) will be a solution to (5): just apply $\partial_{x}^{2}$ on both sides of (9). So, our goal is to produce a solution to (9) for $|\epsilon|<<1$.

Our next result states that one can perturbatively produce a solution of (9) starting from a solution of (8). It is important to note that for the existence of these waves, an important spectral condition involving the linearized operator

$$
L:=-\beta \partial_{x}^{2}+c-f^{\prime}\left(\varphi_{0}\right)
$$

is required. Namely, we shall need that ${ }^{6}\left\langle L^{-1}[1], 1\right\rangle \neq 0$. The significance of this quantity is well-recognized in the literature; see [9] and Chapter 6.1.2 in [17].

\footnotetext{
${ }^{4}$ We would like to point out, on the other hand, that our results are pretty flexible in that they should be applicable to a wide range of examples, satisfying a set of assumptions.

${ }^{5}$ Note that in principle we obtain linear terms $h+m x$ on the right-hand side. But the cases with $m \neq 0$ are not permissible, due to the periodicity requirements in $[-T, T]$, hence $m=0$.

${ }^{6}$ Even though $L$ has a kernel, the constant function 1 is assumed to be orthogonal to it, so this expression makes sense.
} 
Theorem 1. Let the nonlinearity $f$ be so that $f \in C^{2}\left(\mathbf{R}^{1}\right), f(0)=0$ and the following holds:

1. There is an even, mean zero and smooth solution $\varphi_{0} \in L^{2}$ of (8).

2. $\operatorname{Ker}[L] \subset L_{\text {odd }}^{2}$. That is, the kernel of $L$ is spanned by odd functions.

3. $\left\langle L^{-1}[1], 1\right\rangle \neq 0$.

Then, there exists $\epsilon_{0}>0$, a function $\alpha(\epsilon):\left(-\epsilon_{0}, \epsilon_{0}\right) \rightarrow \mathbf{R}^{1}$, so that for all $\epsilon \epsilon$ $\left(-\epsilon_{0}, \epsilon_{0}\right)$, there is a function $\varphi_{\epsilon} \in H_{0, \text { even }}^{2}$, which is a solution to (9). In addition,

$$
\begin{aligned}
\varphi_{\epsilon} & =\varphi_{0}+\epsilon L^{-1}\left[\partial_{x}^{-2} \varphi_{0}-\frac{\left\langle\partial_{x}^{-2} \varphi_{0}, L^{-1}[1]\right\rangle}{\left\langle 1, L^{-1}[1]\right\rangle}\right]+O\left(\epsilon^{2}\right), \\
\alpha(\epsilon) & =-\epsilon \frac{\left\langle\partial_{x}^{-2} \varphi_{0}, L^{-1}[1]\right\rangle}{\left\langle 1, L^{-1}[1]\right\rangle}+O\left(\epsilon^{2}\right) .
\end{aligned}
$$

Proof. Define $\psi_{0}=\frac{L^{-1}[1]}{\left\|L^{-1}[1]\right\|}$. This is a well-defined element of $H_{\text {even }}^{2}[-T, T]$ by the Fredholm alternative, since $1 \perp \operatorname{Ker}[L]$, which follows from $\operatorname{Ker}[L] \subset L_{\text {odd }}^{2}$.

For $\psi \in H_{0, \text { even }}^{2}$, introduce $F: \mathbf{R}^{1} \times \mathbf{R}^{1} \times H_{0, \text { even }}^{2} \rightarrow L_{\text {even }}^{2}$ defined via

$$
F(\epsilon, \alpha ; \psi)=-\beta\left(\varphi_{0}+\psi\right)^{\prime \prime}+c\left(\varphi_{0}+\psi\right)-\epsilon \partial_{x}^{-2}\left(\varphi_{0}+\psi\right)-f\left(\varphi_{0}+\psi\right)-h-\alpha .
$$

Note that the condition $\psi \in L_{0}^{2}$ allows us to take the operator $\partial_{x}^{-2}$ in the definition of $F$.

Let $P_{\left\{\psi_{0}\right\}^{\perp}}$ be the orthogonal projection on the subspace $\left\{\psi_{0}\right\}^{\perp}=\left\{\psi:\left\langle\psi, \psi_{0}\right\rangle=\right.$ $0\}$. That is,

$$
P_{\left\{\psi_{0}\right\}^{\perp}} f=f-\left\langle f, \psi_{0}\right\rangle \psi_{0} .
$$

We prove our theorem via the Lyapunov-Schmidt procedure. First, $F(0,0 ; 0)=0$.

Next, we show that there is $\psi=\psi(\epsilon, \alpha) \in H_{0, \text { even }}^{2}$, so that the equation

$$
P_{\left\{\psi_{0}{ }^{\perp}\right.} F(\epsilon, \alpha ; \psi)=0
$$

holds true for $(\epsilon, \alpha)$ in some neighborhood of $(0,0)$. As an application of the implicit function theorem for (12), in the spaces indicated above, we will need to show that

$$
D_{\psi}(0,0 ; 0): H_{0, \text { even }}^{2} \rightarrow Y
$$

is an invertible operator, where $Y=P_{\left\{\psi_{0}\right\}^{\perp}}\left[L_{0, \text { even }}^{2}\right] \subset L_{0, \text { even }}^{2}$. We have

$$
D_{\psi}(0,0 ; 0) \tilde{\psi}=P_{\left\{\psi_{0}\right\}^{\perp}}\left[-\beta \tilde{\psi}^{\prime \prime}+c \tilde{\psi}-f^{\prime}\left(\varphi_{0}\right) \tilde{\psi}\right]=P_{\left\{\psi_{0}\right\}^{\perp}} L[\tilde{\psi}] .
$$

Let $g \in Y$ be an arbitrary element and consider $D_{\psi}(0,0 ; 0) \tilde{\psi}=g$. That is,

$$
P_{\left\{\psi_{0}\right\}^{\perp}} L[\tilde{\psi}]=g .
$$

Since $\operatorname{Ker}[L]$ is spanned by odd functions, $L$ is invertible on $L_{\text {even }}^{2}$ and hence $\tilde{\psi}=$ $L^{-1}[g] \in H_{\text {even }}^{2}$ is well defined. It remains to check that $\tilde{\psi}$ has mean value zero. We have by the self-adjointness of $L$ on $L_{\text {even }}^{2}$

$$
\langle\tilde{\psi}, 1\rangle=\left\langle L^{-1}[g], 1\right\rangle=\left\langle g, L^{-1}[1]\right\rangle=\left\|L^{-1}[1]\right\|\left\langle g, \psi_{0}\right\rangle=0,
$$

since $g \in Y=P_{\left\{\psi_{0}\right\}^{\perp}}\left[L_{0, \text { even }}^{2}\right]$. Thus, $\tilde{\psi}$ has mean value zero and the first step of the Lyapunov-Schmidt procedure is justified. That is, there is a function $\psi=\psi(\epsilon, \alpha)$.

Copyright (c) by SIAM. Unauthorized reproduction of this article is prohibited. 
For the second step, we need to resolve the remaining equation, namely,

$$
\left\langle F(\epsilon, \alpha ; \psi(\epsilon, \alpha)), \psi_{0}\right\rangle=0 .
$$

Denoting $f(\epsilon, \alpha)=\left\langle F(\epsilon, \alpha ; \psi(\epsilon, \alpha)), \psi_{0}\right\rangle$, we need to check that the implicit function theorem applies, so that there is a solution $\alpha(\epsilon)$. We have

$$
\frac{\partial f}{\partial \alpha}=\left\langle-\beta\left(\frac{\partial \psi}{\partial \alpha}\right)^{\prime \prime}+c \frac{\partial \psi}{\partial \alpha}-\epsilon \partial_{x}^{-2}\left(\frac{\partial \psi}{\partial \alpha}\right)-f^{\prime}\left(\varphi_{0}+a \psi_{0}+\psi\right) \frac{\partial \psi}{\partial \alpha}-1, \psi_{0}\right\rangle .
$$

Evaluating at $\epsilon=0, \alpha=0$ yields

$$
\begin{aligned}
\left.\frac{\partial f}{\partial \alpha}\right|_{\epsilon=0, \alpha=0} & =\left\langle\left. L \frac{\partial \psi}{\partial \alpha}\right|_{\epsilon=0, \alpha=0}, \psi_{0}\right\rangle-\left\langle 1, \psi_{0}\right\rangle \\
& =\frac{1}{\left\|L^{-1}[1]\right\|}\left\langle\left.\frac{\partial \psi}{\partial \alpha}\right|_{\epsilon=0, \alpha=0}, 1\right\rangle-\left\langle 1, \psi_{0}\right\rangle=-\left\langle 1, \psi_{0}\right\rangle,
\end{aligned}
$$

where we have used the self-adjointness of $L$, the fact that $\psi(\epsilon, \alpha) \in L_{0}^{2}$ (and hence $\left.\left.\frac{\partial \psi}{\partial \alpha}\right|_{\epsilon=0, \alpha=0} \perp 1\right)$. Finally, by the assumptions in the theorem,

$$
\left.\frac{\partial f}{\partial \alpha}\right|_{\epsilon=0, \alpha=0}=-\frac{1}{\left\|L_{+}^{-1}[1]\right\|}\left\langle 1, L^{-1}[1]\right\rangle \neq 0
$$

and hence the existence of $\alpha(\epsilon)$ in a small interval $\left(-\epsilon_{0}, \epsilon_{0}\right)$ is shown.

We now establish the behavior of $\alpha(\epsilon)$ and $\psi_{\epsilon}:=\psi(\epsilon, \lambda(\epsilon))$. We start with the relation

$$
P_{\left\{\psi_{0}\right\}^{\perp}} F(\epsilon, \alpha ; \psi(\epsilon, \alpha))=0 .
$$

Taking a derivative in $\epsilon$ and evaluating at $\epsilon=0, \alpha=0$ yields

$$
P_{\left\{\psi_{0}\right\}^{\perp}}\left[L_{+}\left[\left.\frac{\partial \psi}{\partial \epsilon}\right|_{\epsilon=\alpha=0}\right]-\partial_{x}^{-2} \varphi_{0}\right]=0 .
$$

Since $L\left[\left.\frac{\partial \psi}{\partial \epsilon}\right|_{\epsilon=\alpha=0}\right] \perp \psi_{0}$, it follows that

$$
L\left[\left.\frac{\partial \psi}{\partial \epsilon}\right|_{\epsilon=\alpha=0}\right]=P_{\left\{\psi_{0}\right\}^{\perp}}\left[\partial_{x}^{-2} \varphi_{0}\right] .
$$

The right-hand side is even and hence orthogonal to $\operatorname{Ker}[L]$, whence we can find the unique solution

$$
\left.\frac{\partial \psi}{\partial \epsilon}\right|_{\epsilon=\alpha=0}=L^{-1}\left[P_{\left\{\psi_{0}\right\}^{\perp}}\left(\partial_{x}^{-2} \varphi_{0}\right)\right] .
$$

Similarly, taking derivative with respect to $\alpha$ in (14) and evaluating at $\epsilon=\alpha=0$, we obtain

$$
P_{\left\{\psi_{0}\right\}^{\perp}}\left[L_{+}\left[\left.\frac{\partial \psi}{\partial \alpha}\right|_{\epsilon=\alpha=0}\right]-1\right]=0 .
$$

This implies $L\left[\left.\frac{\partial \psi}{\partial \alpha}\right|_{\epsilon=\alpha=0}\right]=P_{\left\{\psi_{0}\right\}^{\perp}}(1)$ and hence

$$
\left.\frac{\partial \psi}{\partial \alpha}\right|_{\epsilon=\alpha=0}=L^{-1}\left[P_{\left\{\psi_{0}\right\}^{\perp}}(1)\right],
$$

since $P_{\left\{\psi_{0}\right\}^{\perp}}(1)$ is an even function. 
Finally, in order to find $\alpha^{\prime}(0)$, we take a derivative in $\epsilon$ in the equation

$$
F(\epsilon, \alpha(\epsilon), \psi(\epsilon, \alpha(\epsilon))=0
$$

and evaluate at $\epsilon=0$. This yields

$$
L\left[\left.\frac{\partial \psi}{\partial \epsilon}\right|_{\epsilon=\alpha=0}\right]+\alpha^{\prime}(0) L\left[\left.\frac{\partial \psi}{\partial \alpha}\right|_{\epsilon=\alpha=0}\right]-\partial_{x}^{-2}\left[\varphi_{0}\right]=\alpha^{\prime}(0) .
$$

Using (15) and (16), this further simplifies to

$$
-\left\langle\partial_{x}^{-2} \varphi_{0}, \psi_{0}\right\rangle \psi_{0}-\alpha^{\prime}(0)\left\langle 1, \psi_{0}\right\rangle \psi_{0}=0
$$

which results in the formula

$$
\alpha^{\prime}(0)=-\frac{\left\langle\partial_{x}^{-2} \varphi_{0}, \psi_{0}\right\rangle}{\left\langle 1, \psi_{0}\right\rangle}
$$

Here again, we have used the condition that $\left\langle 1, \psi_{0}\right\rangle=\left\langle L^{-1}[1], 1\right\rangle \neq 0$. This allows us to derive the following representation formula for $\psi_{\epsilon}$ :

$$
\begin{aligned}
\psi_{\epsilon}(x) & =\left.\epsilon \frac{\partial \psi}{\partial \epsilon}\right|_{\epsilon=\alpha=0}+\left.\alpha(\epsilon) \frac{\partial \psi}{\partial \alpha}\right|_{\epsilon=\alpha=0}+O\left(\epsilon^{2}\right) \\
& =\epsilon\left[L^{-1}\left[P_{\left\{\psi_{0}\right\}^{\perp}}\left(\partial_{x}^{-2} \varphi_{0}\right)\right]-\frac{\left\langle\partial_{x}^{-2} \varphi_{0}, \psi_{0}\right\rangle}{\left\langle 1, \psi_{0}\right\rangle} L^{-1}\left[P_{\left\{\psi_{0}\right\}^{\perp}}(1)\right]\right]+O\left(\epsilon^{2}\right) \\
& =\epsilon L^{-1}\left(P_{\left\{\psi_{0}\right\}^{\perp}}\left[\partial_{x}^{-2} \varphi_{0}-\frac{\left\langle\partial_{x}^{-2} \varphi_{0}, \psi_{0}\right\rangle}{\left\langle 1, \psi_{0}\right\rangle}\right]\right)+O\left(\epsilon^{2}\right) .
\end{aligned}
$$

Since

$$
\left\langle\partial_{x}^{-2} \varphi_{0}-\frac{\left\langle\partial_{x}^{-2} \varphi_{0}, \psi_{0}\right\rangle}{\left\langle 1, \psi_{0}\right\rangle}, \psi_{0}\right\rangle=\left\langle\partial_{x}^{-2} \varphi_{0}, \psi_{0}\right\rangle-\frac{\left\langle\partial_{x}^{-2} \varphi_{0}, \psi_{0}\right\rangle}{\left\langle 1, \psi_{0}\right\rangle}\left\langle 1, \psi_{0}\right\rangle=0,
$$

we find that $P_{\left\{\psi_{0}\right\}^{\perp}}\left[\partial_{x}^{-2} \varphi_{0}-\frac{\left\langle\partial_{x}^{-2} \varphi_{0}, \psi_{0}\right\rangle}{\left\langle 1, \psi_{0}\right\rangle}\right]=\partial_{x}^{-2} \varphi_{0}-\frac{\left\langle\partial_{x}^{-2} \varphi_{0}, \psi_{0}\right\rangle}{\left\langle 1, \psi_{0}\right\rangle}$, and so we finally have the formula

$$
\psi_{\epsilon}(x)=\epsilon L^{-1}\left[\partial_{x}^{-2} \varphi_{0}-\frac{\left\langle\partial_{x}^{-2} \varphi_{0}, \psi_{0}\right\rangle}{\left\langle 1, \psi_{0}\right\rangle}\right]+O\left(\epsilon^{2}\right) .
$$

2.1. Examples of periodic traveling wave solutions of $\mathrm{KdV}$ satisfying Theorem 1. As we have mentioned above, we consider only the KdV case. That is, take $f(z)=z^{2}$. Integrating once more (9), we get the equation

$$
\varphi^{\prime 2}=\frac{2}{3 \beta}\left(-\varphi^{3}+\frac{3}{2} c \varphi^{2}+3 h \varphi+3 h_{2}\right) .
$$

Let $c>0$ and $\varphi_{1}<\varphi_{2}<\varphi_{3}$ are roots of the polynomial $F(\rho)=-\rho^{3}+\frac{3}{2} c \rho^{2}+3 h \rho+3 h_{2}$. Then, we have $F(\rho)=\left(\rho-\varphi_{1}\right)\left(\rho-\varphi_{2}\right)\left(\varphi_{3}-\rho\right)$ and

$$
\mid \begin{aligned}
& \varphi_{1}+\varphi_{2}+\varphi_{3}=\frac{3}{2} c \\
& \varphi_{1} \varphi_{2}+\varphi_{1} \varphi_{3}+\varphi_{2} \varphi_{3}=-3 h \\
& \varphi_{1} \varphi_{2} \varphi_{3}=3 h_{2} .
\end{aligned}
$$

Copyright $($ C by SIAM. Unauthorized reproduction of this article is prohibited. 
Introducing a new variable $s$ via $\varphi=\varphi_{2}+\left(\varphi_{3}-\varphi_{2}\right) s^{2}$, we get

$$
s^{\prime 2}=\frac{1}{6 \beta}\left(1-s^{2}\right)\left(\kappa^{\prime 2}+\kappa^{2} s^{2}\right)
$$

and the solution of (18) is given by

$$
\varphi(x)=\varphi_{2}+\left(\varphi_{3}-\varphi_{2}\right) c n^{2}(\alpha x, \kappa),
$$

where

$$
\kappa^{2}=\frac{2 \varphi_{3}-2 \varphi_{2}}{4 \varphi_{3}+2 \varphi_{2}-3 c}, \quad \kappa^{\prime 2}=1-\kappa^{2}, \quad \alpha^{2}=\frac{4 \varphi_{3}+2 \varphi_{2}-3 c}{12 \beta} .
$$

For fixed $T$ in a proper interval, we can determine $\varphi_{2}$ and $\varphi_{3}$ as smooth functions of $c$ so that the periodic solution $\varphi$ given by (20) will have period $T$ because of monotonicity of the period (for more details see [1, 12]). Moreover, for $T>0$ and $c>0$ there exists a smooth branch of cnoidal waves with mean zero (see [1]).

In $[-T, T]=[-K(k) / \alpha, K(k) / \alpha]$, we consider the spectral properties of the operator

$$
L=-\beta \partial_{x}^{2}+c-2 \varphi,
$$

supplied with periodic boundary conditions. By the above formulas, $\varphi_{3}-\varphi_{2}=$ $6 \beta \alpha^{2} k^{2}, 2 \varphi_{2}-c=4 \beta \alpha^{2}\left(1-2 k^{2}\right)$. Taking $y=\alpha x$ as an independent variable in $L_{+}$, one obtains $L_{+}=\beta \alpha^{2} \Lambda$ with an operator $\Lambda$ in $[-K(k), K(k)]$ given by

$$
\Lambda=-\partial_{x}^{2}-4\left(1+k^{2}\right)+12 k^{2} s n^{2}(y ; k) .
$$

The spectral properties of the operator $\Lambda$ in $[0,2 K(k)]$ are well known. The first three (simple) eigenvalues and corresponding eigenfunctions of $\Lambda$ are

$$
\begin{aligned}
\mu_{0} & =k^{2}-2-2 \sqrt{1-k^{2}+4 k^{4}}<0, \\
\psi_{0}(y) & =d n(y ; k)\left[1-\left(1+2 k^{2}-\sqrt{1-k^{2}+4 k^{4}}\right) \operatorname{sn}^{2}(y ; k)\right]>0, \\
\mu_{1} & =0, \\
\psi_{1}(y) & =d n(y ; k) \operatorname{sn}(y ; k) \operatorname{cn}(y ; k)=\frac{1}{2} \frac{d}{d y} \operatorname{cn}^{2}(y ; k), \\
\mu_{2} & =k^{2}-2+2 \sqrt{1-k^{2}+4 k^{4}}>0, \\
\psi_{2}(y) & =d n(y ; k)\left[1-\left(1+2 k^{2}+\sqrt{1-k^{2}+4 k^{4}}\right) \operatorname{sn}^{2}(y ; k)\right] .
\end{aligned}
$$

Since the eigenvalues of $L$ and $\Lambda$ are related by $\lambda_{n}=\beta \alpha^{2} \mu_{n}$, it follows that the first three eigenvalues of the operator $L$, equipped with periodic boundary condition on $[0,2 K(k)]$ are simple and $\lambda_{0}<0, \lambda_{1}=0, \lambda_{2}>0$. The corresponding eigenfunctions are $\psi_{0}(\alpha x), \psi_{1}(\alpha x)=\varphi^{\prime}$ and $\psi_{2}(\alpha x)$.

Now we verify that condition 3 of Theorem 1 is satisfied in this case, for some interval of values of $\kappa$. We will proceed as in [9]. We have $L \varphi^{\prime}=0$. The function

$$
\psi(x)=\varphi^{\prime}(x) \int^{x} \frac{1}{\varphi^{\prime 2}(s)} d s,\left|\begin{array}{cc}
\varphi^{\prime} & \psi \\
\varphi^{\prime \prime} & \psi^{\prime}
\end{array}\right|=1,
$$

is also solution of $L_{+} \psi=0$. Formally, since $\varphi^{\prime}$ has two zeros using the identities

$$
\frac{1}{c n^{2}(y, \kappa)}=\frac{1}{d n(y, \kappa)} \frac{\partial}{\partial_{y}} \frac{s n(x, \kappa)}{c n(y, \kappa)}, \frac{1}{s n^{2}(y, \kappa)}=-\frac{1}{d n(y, \kappa)} \frac{\partial}{\partial_{y}} \frac{c n(x, \kappa)}{s n(y, \kappa)}
$$

Copyright (c) by SIAM. Unauthorized reproduction of this article is prohibited. 
and integrating by parts we get

$$
\begin{aligned}
\psi(x)= & \frac{1}{2 \alpha^{2}\left(\varphi_{3}-\varphi_{2}\right)}\left[c n^{2}(\alpha x, \kappa)-\frac{1}{1-\kappa^{2}} s n^{2}(\alpha x, \kappa)\right] \\
& -\frac{\kappa^{2} s n(\alpha x, \kappa) c n(\alpha x, \kappa) d n(\alpha x, \kappa)}{2 \alpha\left(\varphi_{3}-\varphi_{2}\right)\left(1-\kappa^{2}\right)} \int_{0}^{x} \frac{\left(1-2 \kappa^{2}\right)-\left(2-\kappa^{2}\right) s n^{2}(\alpha s, \kappa)}{d n^{2}(\alpha s, \kappa)} d s .
\end{aligned}
$$

Thus, we may construct the Green function

$$
L^{-1} f=\varphi^{\prime} \int_{0}^{x} \psi(s) f(s) d s-\psi(s) \int_{0}^{x} \varphi^{\prime}(s) f(s) s+C_{f} \psi(x)
$$

where $C_{f}$ is chosen such that $L^{-1} f$ is periodic with the same period as $\varphi(x)$. After integrating by parts, we get

$$
\left\langle L^{-1}[1], 1\right\rangle=\left(\varphi(T)+\varphi(0)+C_{1}\right) \int_{-T}^{T} \psi(x) d x-2 \int_{-T}^{T} \varphi(x) \psi(x) d x,
$$

where

$$
C_{1}=(\varphi(T)-\varphi(0))-\frac{\varphi^{\prime \prime}(T)}{2 \psi(T)} \int_{-T}^{T} \psi(x) d x
$$

Using that

$$
\operatorname{sn}(\alpha x, \kappa) \operatorname{cn}(\alpha x, \kappa) d n(\alpha x, \kappa)=-\frac{1}{2 \alpha \kappa^{2}} \frac{\partial}{\partial x} d n^{2}(\alpha x, \kappa)
$$

and integrating by parts, we get

$$
\int_{-T}^{T} \psi(x) d x=\frac{1}{\alpha^{3}\left(\varphi_{3}-\varphi_{2}\right)} I_{1}
$$

where

$$
\begin{aligned}
I_{1}(\kappa)= & \int_{0}^{K(\kappa)} c n^{2}(x, \kappa) d x-\frac{1}{1-\kappa^{2}} \int_{0}^{K(\kappa)} s n^{2}(x, \kappa) d x \\
& +\frac{1}{2\left(1-\kappa^{2}\right)} \int_{0}^{K(\kappa)} \frac{\left[\left(1-2 \kappa^{2}\right)-\left(2-\kappa^{2}\right) s n^{2}(x, \kappa)\right]\left[\left(1-\kappa^{2}\right)-d n^{2}(x, \kappa)\right]}{d n^{2}(x, \kappa)} d x .
\end{aligned}
$$

Using Mathematica, we obtain the following simple formula for $I_{1}(\kappa)$ :

$$
I_{1}(\kappa)=\frac{\left(\kappa^{4}+5 \kappa^{2}-8\right) E(\kappa)+\left(2 \kappa^{4}-9 \kappa^{2}+8\right) K(\kappa)}{2\left(k^{4}-k^{2}\right)} .
$$

Now

$$
\int_{-T}^{T} \varphi(x) \psi(x) d x=\frac{\varphi_{2}}{\alpha^{3}\left(\varphi_{3}-\varphi_{2}\right)} I_{1}+\left(\varphi_{3}-\varphi_{2}\right) \int_{-T}^{T} c n^{2}(\alpha x, \kappa) \psi(x) d x .
$$

Using that

$$
c n^{3}(\alpha x, \kappa) \operatorname{sn}(\alpha x, \kappa) d n(\alpha x, \kappa)=-\frac{1}{4 \alpha} \frac{\partial}{\partial x} c n^{4}(\alpha x, \kappa)
$$

Copyright (c) by SIAM. Unauthorized reproduction of this article is prohibited. 
and integrating by parts, we get

$$
\int_{-T}^{T} c n^{2}(\alpha x, \kappa) \psi(x) d x=\frac{1}{\alpha^{3}\left(\varphi_{3}-\varphi_{2}\right)} I_{2}
$$

where

$$
\begin{aligned}
I_{2}= & \int_{0}^{K(\kappa)} c n^{4}(x, \kappa) d x-\frac{1}{1-\kappa^{2}} \int_{0}^{K(\kappa)} s n^{2}(x, \kappa) c n^{2}(x, \kappa) d x \\
& -\frac{\kappa^{2}}{4\left(1-\kappa^{2}\right)} \int_{0}^{K(\kappa)} \frac{c n^{4}(x, \kappa)\left[\left(1-2 \kappa^{2}\right)-\left(2-\kappa^{2}\right) s n^{2}(x, \kappa)\right]}{d n^{2}(x, \kappa)} d x .
\end{aligned}
$$

Using Mathematica, we obtain the following formula for $I_{2}(\kappa)$ :

$$
I_{2}(\kappa)=\frac{2 E(\kappa)-K(\kappa)}{4} .
$$

Hence

$$
\left\langle L^{-1}[1], 1\right\rangle=\frac{T^{3}}{K^{3}(\kappa)}\left[\frac{\varphi(T)+\varphi(0)+C_{1}}{\varphi_{3}-\varphi_{2}} I_{1}-\frac{2 \varphi_{2}}{\varphi_{3}-\varphi_{2}} I_{1}-2 I_{2}\right],
$$

where we used that $T=\frac{K(\kappa)}{\alpha}$. From (19)-(21), after calculations, we obtain

$$
\begin{aligned}
& \varphi_{3}-\varphi_{2}=6 \beta \kappa^{2} \frac{K^{2}(\kappa)}{T^{2}}, \\
& \varphi_{2}=-\frac{6 \beta}{T^{2}} K(\kappa)\left[E(\kappa)-\left(1-\kappa^{2}\right) K(\kappa),\right. \\
& \varphi_{3}=\frac{6 \beta}{T^{2}}\left[K^{2}(\kappa)-E(\kappa) K(\kappa)\right], \\
& c=\frac{4 \beta}{T^{2}}\left[\left(2-\kappa^{2}\right) K^{2}(\kappa)-3 E(\kappa) K(\kappa)\right] .
\end{aligned}
$$

Note that the wave speed $c$ needs to be a positive quantity by construction. On the other hand, this is not always satisfied - indeed, it is only true for some values of $\kappa$; refer to Figure 1. It is clear that only values of $\kappa \in(0.98,1)$ produce $c=c(\kappa)>0$ as is required. From (25), we get

$$
\frac{\varphi(T)+\varphi(0)+C_{1}}{\varphi_{3}-\varphi_{2}}=\frac{2\left(1-\kappa^{2}\right) K(\kappa)-2 E(\kappa)-\frac{2\left(1-\kappa^{2}\right) K(\kappa) I_{1}}{\int_{0}^{K(\kappa)} \frac{\left(1-2 \kappa^{2}\right)-\left(2-\kappa^{2}\right) s n^{2}(x, \kappa)}{d n^{2}(x, \kappa)} d x}}{\kappa^{2} K(\kappa)}
$$

and

$$
\frac{\varphi_{2}}{\varphi_{3}-\varphi_{2}}=\frac{\left(1-\kappa^{2}\right) K(\kappa)-E(\kappa)}{\kappa^{2} K(\kappa)} .
$$

Using Mathematica, we were able to simplify the formula (24) to the following: $\left\langle L^{-1}[1], 1\right\rangle=\frac{T^{3}}{K^{3}(\kappa)} Z(\kappa)$, where

$$
\begin{aligned}
Z(\kappa)= & \frac{\left(3 \kappa^{8}-33 \kappa^{6}+111 \kappa^{4}-144 \kappa^{2}+64\right) K(\kappa)^{2}+2\left(4 \kappa^{8}-3 \kappa^{6}-50 \kappa^{4}+112 \kappa^{2}-64\right) K(\kappa) E(\kappa)}{2 \kappa^{4}\left(2\left(\kappa^{4}-\kappa^{2}+1\right) E(\kappa)-\left(\kappa^{4}-3 \kappa^{2}+2\right) K(\kappa)\right)} \\
& +\frac{\left(-3 \kappa^{8}+14 \kappa^{6}+5 \kappa^{4}-80 \kappa^{2}+64\right) E(\kappa)^{2}}{\left(2\left(\kappa^{4}-\kappa^{2}+1\right) E(\kappa)-\left(\kappa^{4}-3 \kappa^{2}+2\right) K(\kappa)\right)} .
\end{aligned}
$$

We have checked, by plotting in Mathematica, that in the interval of interest $\kappa \in$ $(0.98,1)$ (i.e., the interval in which $c>0)$, the function $Z[\kappa]>0, \kappa \in(0.98,1)$, whence $\left\langle L^{-1}[1], 1\right\rangle>0$.

Copyright $@$ by SIAM. Unauthorized reproduction of this article is prohibited. 


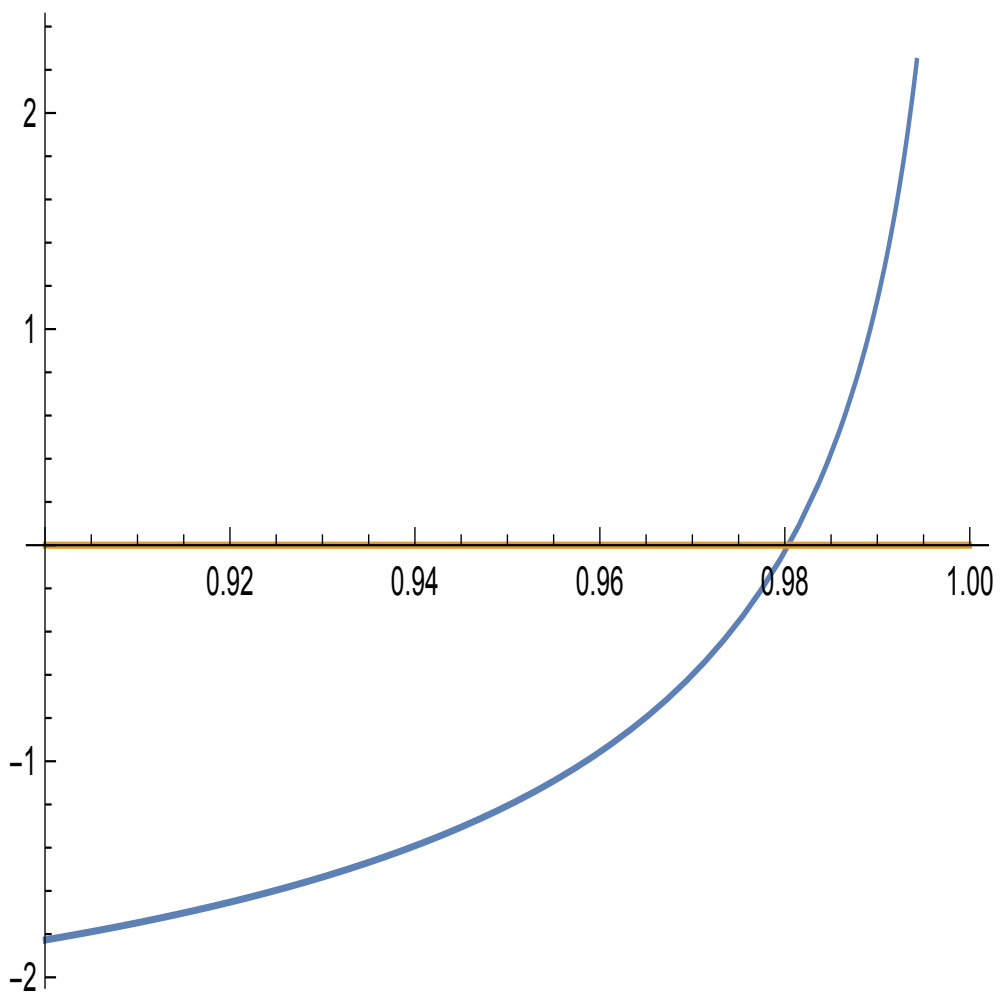

FIG. 1. The graph of the function $\left(2-\kappa^{2}\right) K^{2}(\kappa)-3 E(\kappa) K(\kappa)$ for $\kappa \in(0.9,1)$.

3. Stability of traveling waves of the RSPE. We study the linear stability of the waves $\varphi_{\epsilon}$ with respect of perturbations of the same period. We assume that these solutions are even functions of $x$.

3.1. Spectral setup. Consider the linearization around the solution $\varphi_{\epsilon}(x-c t)$ of (2), namely, $u(t, x)=\varphi_{\epsilon}(x-c t)+v(t, x-c t)$. Note that since all solutions must have mean value zero, we take the perturbation $v \in L_{0}^{2}[-T, T]$ as well. After ignoring all terms of the form $O\left(v^{2}\right)$, we obtain

$$
v_{t x}=\left(-\beta v_{x x}+c v-f^{\prime}\left(\varphi_{\epsilon}\right) v-\epsilon \partial_{x}^{-2} v\right)_{x x} .
$$

By the mean value property of $v$, we can apply the operator $\partial_{x}^{-1}$ to the previous identity to obtain

$$
v_{t}=\left(-\beta v_{x x}+c v-f^{\prime}\left(\varphi_{\epsilon}\right) v-\epsilon \partial_{x}^{-2} v\right)_{x} .
$$

Introduce the one-parameter family of operators

$$
\mathcal{L}_{\epsilon}:=-\beta \partial_{x x}+c-f^{\prime}\left(\varphi_{\epsilon}\right)-\epsilon \partial_{x}^{-2} .
$$

We denote the standard Hill operator corresponding to $\epsilon=0$ by $L$, that is, $L:=\mathcal{L}_{0}$. Clearly, $\mathcal{L}_{\epsilon}$ is still self-adjoint, with domain $H_{0}^{2}$, which acts invariantly on the even and odd subspaces.

In order to rewrite the linear stability problem (26) in a more suitable form, introduce

$$
w=\left|\partial_{x}\right|^{-1 / 2} v, w \in H_{0}^{5 / 2} .
$$

Copyright $@$ by SIAM. Unauthorized reproduction of this article is prohibited. 
We then have, in terms of $w$ (recall $\left.\partial_{x}=-H\left|\partial_{x}\right|\right)$,

$$
\left|\partial_{x}\right|^{1 / 2} w_{t}=H\left|\partial_{x}\right| \mathcal{L}_{\epsilon}\left|\partial_{x}\right|^{1 / 2} w
$$

Applying $\left|\partial_{x}\right|^{-1 / 2}$ on both sides (and taking into account that $H$ commutes with all $\left.\left|\partial_{x}\right|^{s}\right)$, we obtain

$$
w_{t}=-H\left|\partial_{x}\right|^{1 / 2} \mathcal{L}_{\epsilon}\left|\partial_{x}\right|^{1 / 2} w=:-H \mathcal{K}_{\epsilon} w .
$$

Setting $w(t, x) \rightarrow e^{\lambda t} w(x)$, we arrive at the eigenvalue problem

$$
\lambda w=-H \mathcal{K}_{\epsilon} w .
$$

Note that $H^{*}=-H$, while $\mathcal{K}_{\epsilon}^{*}=\mathcal{K}_{\epsilon}$. This is exactly the form of the eigenvalue problem considered in $[15,16]$. It is a standard result that the essential spectrum of (28) is on the imaginary axes and the instabilities may come only in the form of point eigenvalues $\lambda$ with positive real part. In order to decide about the stability of (28), we need to establish some properties of the operators $\mathcal{L}_{\epsilon}, \mathcal{K}_{\epsilon}$. To begin with, observe that $\mathcal{K}_{\epsilon}$ is a self-adjoint operator with a domain $H_{p e r}^{2}[-T, T]$.

3.2. Spectral analysis. We need the following simple lemma.

Lemma 1. Assume that the operator $L$ has one simple negative eigenvalue, a simple eigenvalue at zero, corresponding to the eigenfunction $\varphi_{0}^{\prime}$ and the rest of the spectrum is strictly inside $(0, \infty)$. In addition, we require that $\left\langle L^{-1}[1], 1\right\rangle \neq 0$.

Then, there exists $\epsilon_{1}>0$, so that for all $\epsilon \in\left(-\epsilon_{1}, \epsilon_{1}\right)$, the self-adjoint operator $\mathcal{K}_{\epsilon}$ is either a nonnegative operator or else it has one simple negative eigenvalue. In addition, it has a simple eigenvalue at zero, corresponding to the eigenfunction $q_{\epsilon}=H\left|\partial_{x}\right|^{1 / 2} \varphi_{\epsilon}$, while the rest of the spectrum belongs to $(0, \infty)$.

Proof. We start our considerations with the operators $\mathcal{L}_{\epsilon}$. We have that

$$
\mathcal{L}_{\epsilon}=\mathcal{L}_{0}+f^{\prime}\left(\varphi_{0}\right)-f^{\prime}\left(\varphi_{\epsilon}\right)-\epsilon \partial_{x}^{-2}=L+M_{\epsilon},
$$

where $M_{\epsilon}$ is clearly a bounded operator, with $\left\|M_{\epsilon}\right\|_{L^{2} \rightarrow L^{2}} \leq C \epsilon$. Thus, by the Courant principle for the eigenvalues, ${ }^{7}$ we can conclude that $\left|\lambda_{j}\left(\mathcal{L}_{\epsilon}\right)-\lambda_{j}(L)\right| \leq C \epsilon$.

Thus, for all small enough $\epsilon$, we have one negative eigenvalue for $\mathcal{L}_{\epsilon}$ (which is close to $\left.\lambda_{0}(L)<0\right)$. The eigenvalue $\lambda_{2}\left(\mathcal{L}_{\epsilon}\right)$ is in fact positive, being close to $\lambda_{2}(L)>0$. The only remaining question is then about the sign of $\lambda\left(\mathcal{L}_{\epsilon}\right)$, which is close to $\lambda_{1}(L)=0$. By direct verification (differentiating (9) in $x$ ), we have that $\mathcal{L}_{\epsilon}\left(\varphi_{\epsilon}^{\prime}\right)=0,0$ is an eigenvalue, and thus that $\lambda_{1}\left(\mathcal{L}_{\epsilon}\right)=0$. To summarize,

$$
\lambda_{0}\left(\mathcal{L}_{\epsilon}\right)<0=\lambda_{1}\left(\mathcal{L}_{\epsilon}\right)<\lambda_{2}\left(\mathcal{L}_{\epsilon}\right) .
$$

We now need to make similar arguments regarding $\mathcal{K}_{\epsilon}$. Let $\psi_{\epsilon}$ be the negative eigenfunction for $\mathcal{L}_{\epsilon}$. According to the Courant variational eigenvalue principle, we have

$$
\lambda_{1}\left(\mathcal{K}_{\epsilon}\right) \geq \inf _{\phi \perp\left|\partial_{x}\right|^{1 / 2} \psi_{\epsilon}} \frac{\left\langle\mathcal{K}_{\epsilon} \phi, \phi\right\rangle}{\|\phi\|^{2}} .
$$

The restriction in the inf is equivalent to $\left\langle\left|\partial_{x}\right|^{1 / 2} \phi, \psi_{\epsilon}\right\rangle=0$. But then,

$$
\left\langle\mathcal{K}_{\epsilon} \phi, \phi\right\rangle=\left\langle\mathcal{L}_{\epsilon}\left|\partial_{x}\right|^{1 / 2} \phi,\left|\partial_{x}\right|^{1 / 2} \phi\right\rangle \geq 0,
$$

\footnotetext{
$\lambda_{0}(S)$.

${ }^{7}$ Here,$\lambda_{j}(S)$ refers to the $j$ th eigenvalue of the self-adjoint operator $S$, the smallest one being
} Copyright (C) by SIAM. Unauthorized reproduction of this article is prohibited. 
since $\left.\mathcal{L}_{\epsilon}\right|_{\left\{\psi_{\epsilon}\right\}^{\perp}} \geq 0$. Thus, we conclude that $\lambda_{1}\left(\mathcal{K}_{\epsilon}\right) \geq 0$. This last conclusion implies that either $\mathcal{K}_{\epsilon} \geq 0$ or else it has at most one simple and negative eigenvalue.

Next, we study $\operatorname{Ker}\left(\mathcal{K}_{\epsilon}\right)$. We will show that it is a one-dimensional subspace, spanned by $q_{\epsilon}=H\left|\partial_{x}\right|^{1 / 2} \varphi_{\epsilon}$. To that end, let $\mathcal{K}_{\epsilon} \phi=\left|\partial_{x}\right|^{1 / 2} \mathcal{L}_{\epsilon}\left|\partial_{x}\right|^{1 / 2} \phi=0$. As a consequence

$$
\mathcal{L}_{\epsilon}\left|\partial_{x}\right|^{1 / 2} \phi=c
$$

where $c$ could be zero or a nonzero constant. We show that it must be that $c=0$ for all small enough $\epsilon$. Indeed, assume for a moment that $c \neq 0$. Then, since $c \perp$ $\operatorname{Ker}\left(\mathcal{L}_{\epsilon}\right)=\operatorname{span}\left\{\varphi_{\epsilon}^{\prime}\right\}$, we can take inverses in (29) and

$$
\left|\partial_{x}\right|^{1 / 2} \phi=c \mathcal{L}_{\epsilon}^{-1}[1]
$$

But now, take a dot product of this last expression with the constant 1. We have

$$
0=\left\langle\left|\partial_{x}\right|^{1 / 2} 1, \phi\right\rangle=\left\langle 1,\left|\partial_{x}\right|^{1 / 2} \phi\right\rangle=c\left\langle 1, \mathcal{L}_{\epsilon}^{-1}[1]\right\rangle .
$$

Since $\lim _{\epsilon \rightarrow 0}\left\langle 1, \mathcal{L}_{\epsilon}^{-1}[1]\right\rangle=\left\langle 1, L^{-1}[1]\right\rangle \neq 0$, it follows that $c=0$, a contradiction. Thus, $\mathcal{L}_{\epsilon}\left|\partial_{x}\right|^{1 / 2} \phi=0$. However, recall that $\mathcal{L}_{\epsilon}$ has unique eigenfunction at zero, $\varphi_{\epsilon}^{\prime}$. Thus, $\left|\partial_{x}\right|^{1 / 2} \phi=$ const. $\partial_{x} \varphi_{\epsilon}$ or

$$
\phi=\text { const. }\left|\partial_{x}\right|^{-1 / 2} \partial_{x} \varphi_{\epsilon}=\text { const. } H\left|\partial_{x}\right|^{1 / 2} \varphi_{\epsilon} .
$$

Finally, we need to show that the rest of the spectrum of $\mathcal{K}_{\epsilon}$ is in $(0, \infty)$. Indeed, we have already shown that $\lambda_{1}\left(\mathcal{K}_{\epsilon}\right)=0$. If $\lambda_{2}\left(\mathcal{K}_{\epsilon}\right)>0$, we are done. Otherwise, we would have $\lambda_{2}\left(\mathcal{K}_{\epsilon}\right)=0$, which means that zero is an eigenvalue of multiplicity of at least two for $\mathcal{K}_{\epsilon}$. We have of course ruled this out in the argument above, so $\lambda_{2}\left(\mathcal{K}_{\epsilon}\right)>0$ and Lemma 1 is proved in full.

For the stability of the waves, we observe that the eigenvalue problems that we have to deal with are in the form considered in $[15,16]$. In these papers, the authors have studied a pretty general form of eigenvalue problems associated to a linearization around soliton solutions of Hamiltonian systems. According to Lemma 1, we have that $\mathcal{K}_{\epsilon}$ is either a positive operator or else it has one negative and simple eigenvalue. If $\mathcal{K}_{\epsilon} \geq 0$, we have that ${ }^{8} n\left(\mathcal{K}_{\epsilon}\right)=0$ and hence, according to the results ${ }^{9}$ of $[15,16]$ the wave is stable. Otherwise, $n\left(\mathcal{K}_{\epsilon}\right)=1$ and we have by [16] that the number of instabilities of (28) - that is, the number of solutions $(\lambda, w): \Re \lambda>0, w \in D\left(\mathcal{K}_{\epsilon}\right)$ of (28) - is equal to

$$
n\left(\mathcal{K}_{\epsilon}\right)-n\left(\left\langle\mathcal{K}_{\epsilon}^{-1} H q_{\epsilon}, H q_{\epsilon}\right\rangle\right)=1-n\left(\left\langle\mathcal{K}_{\epsilon}^{-1} H q_{\epsilon}, H q_{\epsilon}\right\rangle\right) .
$$

Thus, we need to compute the quantity

$$
\left\langle\mathcal{K}_{\epsilon}^{-1} H q_{\epsilon}, H q_{\epsilon}\right\rangle=\left\langle\left|\partial_{x}\right|^{-1 / 2} \mathcal{L}_{\epsilon}^{-1}\left|\partial_{x}\right|^{-1 / 2}\left[\left|\partial_{x}\right|^{1 / 2} \varphi_{\epsilon}\right],\left|\partial_{x}\right|^{1 / 2} \varphi_{\epsilon}\right\rangle=\left\langle\mathcal{L}_{\epsilon}^{-1} \varphi_{\epsilon}, \varphi_{\epsilon}\right\rangle .
$$

Since both $\mathcal{L}_{\epsilon}$ and $L$ act invariantly and are invertible on the subspace of even functions, we take the operator norms in the space of $L_{\text {even }}^{2}$. We have

$$
\left\|\mathcal{L}_{\epsilon}^{-1}-L^{-1}\right\|=\left\|\left(\left(I+L^{-1} M_{\epsilon}\right)^{-1}-I\right) L^{-1}\right\| \leq\left\|L^{-1}\right\| \|\left(\left(I+L^{-1} M_{\epsilon}\right)^{-1}-I \| .\right.
$$

\footnotetext{
${ }^{8}$ Here $n(S)$ denotes the number of negative eigenvalues of the self-adjoint operator $S$.

${ }^{9}$ In this simple case, one in fact does not need the results of $[15,16]$; one can pretty much rule out instabilities by an easy hands-on argument.
} 
It follows that $\left\|\mathcal{L}_{\epsilon}^{-1}-L^{-1}\right\| \leq C \epsilon$. Furthermore, $\left\|\varphi_{\epsilon}-\varphi\right\| \leq C \epsilon$ and hence we have that

$$
\lim _{\epsilon \rightarrow 0}\left\langle\mathcal{L}_{\epsilon}^{-1} \varphi_{\epsilon}, \varphi_{\epsilon}\right\rangle=\left\langle L^{-1} \varphi, \varphi\right\rangle .
$$

We summarize the existence and stability findings for $\varphi_{\epsilon}$ in the following result.

TheOREM 2. Assume that the nonlinearity $f$, the even solution $\varphi$ of (8), and the operator $L=-\beta \partial_{x}^{2}+c-f^{\prime}(\varphi)$ satisfy the assumptions of Theorem 1 . Then, there exists $\epsilon_{0}>0$, so that traveling waves $\varphi_{\epsilon}$ exist for all $\epsilon \in\left(-\epsilon_{0}, \epsilon_{0}\right)$. The functions $\varphi_{\epsilon}$ are even.

Furthermore, assume that $L$ has a simple and single negative e-value and a simple eigenvalue at zero (with kernel spanned by $\varphi^{\prime}$ ). Then, under the assumption

$$
\left\langle L^{-1}[\varphi], \varphi\right\rangle<0
$$

we can conclude that all waves $\varphi_{\epsilon}$ are linearly stable when perturbed with perturbations with the same period.

Note that (30) is nothing but the Vakhitov-Kolokolov stability criterion, according to the Hamilton-Krein index theory. By way of example, we examine the properties of the periodic traveling wave solutions of $\mathrm{KdV}$, constructed in section 2.1. Suppose $\varphi$ is the periodic traveling wave for KdV provided in (20). Then, by Theorem 1, there exists $\epsilon_{0}>0$, so that for all $\epsilon:|\epsilon|<\epsilon_{0}$, there exist traveling wave solutions $\varphi_{\epsilon}$ of the RSPE. By checking again the properties of $\varphi$, we conclude that these are spectrally stable, according to Theorem 2 . We can thus formulate the following corollary.

Corollary 1. Let $\varphi$ be given (20) with $\kappa \in(0.98,1)$. Then, for all $\epsilon:|\epsilon|<<1$, the corresponding traveling waves $\varphi_{\epsilon}$ of the regularized SPE are spectrally stable.

Unfortunately, while spectral stability is established via the instabilities index counting methods, we cannot say anything definite about orbital stability.

Proof. In order to establish the stability of the waves, we must show that $\left\langle L^{-1} \varphi, \varphi\right\rangle<0$. Thus, we need to compute $\left\langle L^{-1} \varphi, \varphi\right\rangle$. To that end, we start with $L[1]=c-2 \varphi$. Applying $L^{-1}$ yields

$$
1=c L^{-1}[1]-2 L^{-1} \varphi
$$

Since $\varphi$ has mean value zero, we conclude

$$
\left\langle L^{-1} \varphi, \varphi\right\rangle=\frac{c}{2}\left\langle L^{-1}[1], \varphi\right\rangle .
$$

Integrating by parts, we get

$$
\begin{gathered}
\left\langle L^{-1}[1], \varphi\right\rangle=\frac{\varphi^{2}(T)}{2} \int_{-T}^{T} \psi(x) d x-\frac{3}{2} \int_{-T}^{T} \varphi^{2}(x) \psi(x) d x+\left[\varphi(0)+C_{1}\right] \int_{-T}^{T} \varphi(x) \psi(x) d x \\
=\frac{\varphi^{2}(T)}{2 \alpha^{3}\left(\varphi_{3}-\varphi_{2}\right)} I_{1}+\left[\varphi(0)+C_{1}\right]\left[\frac{\varphi_{2}}{\alpha^{3}\left(\varphi_{3}-\varphi_{2}\right)} I_{1}+\frac{1}{\alpha^{3}} I_{2}\right]-\frac{3}{2} \int_{-T}^{T} \varphi^{2}(x) \psi(x) d x
\end{gathered}
$$

and

$$
\int_{-T}^{T} \varphi^{2}(x) \psi(x) d x=\frac{\varphi_{2}^{2}}{\alpha^{3}\left(\varphi_{3}-\varphi_{2}\right)} I_{1}+\frac{2 \varphi_{2}}{\alpha^{3}} I_{2}+\left(\varphi_{3}-\varphi_{2}\right)^{2} \int_{-T}^{T} c n^{4}(\alpha x, \kappa) \psi(x) d x .
$$

Copyright (c) by SIAM. Unauthorized reproduction of this article is prohibited. 
Using the identity

$$
c n^{5}(\alpha x, \kappa) \operatorname{sn}(\alpha x, \kappa) d n(\alpha x, \kappa)=-\frac{1}{6 \alpha} \frac{\partial}{\partial x} c n^{6}(\alpha x, \kappa)
$$

and integrating by parts, we get

$$
\int_{-T}^{T} c n^{4}(\alpha x, \kappa) \psi(x) d x=\frac{1}{\alpha^{3}\left(\varphi_{3}-\varphi_{2}\right)} I_{3}
$$

where

$$
\begin{aligned}
I_{3}(\kappa)= & \int_{0}^{K(\kappa)} c n^{6}(x, \kappa) d x-\frac{1}{1-\kappa^{2}} \int_{0}^{K(\kappa)} s n^{2}(x, \kappa) c n^{4}(x, \kappa) d x \\
& -\frac{\kappa^{2}}{6\left(1-\kappa^{2}\right)} \int_{0}^{K(\kappa)} \frac{c n^{6}(x, k)\left[\left(1-2 \kappa^{2}\right)-\left(2-\kappa^{2}\right) s n^{2}(x, k)\right]}{d n^{2}(x, \kappa)} d x .
\end{aligned}
$$

We have used Mathematica to compute the precise value of $I_{3}(\kappa)$ and we have found

$$
I_{3}(\kappa)=\frac{\left(2 \kappa^{2}-1\right) E(\kappa)+\left(1-\kappa^{2}\right) K(\kappa)}{6 \kappa^{2}} .
$$

Combining the above equalities, we get

$$
\begin{aligned}
\left\langle L^{-1}[1], \varphi\right\rangle= & \frac{1}{\alpha^{3}}\left(\frac{\varphi^{2}(T)}{2\left(\varphi_{3}-\varphi_{2}\right)}+\frac{\left[\varphi(0)+C_{1}\right] \varphi_{2}}{\varphi_{3}-\varphi_{2}}-\frac{3 \varphi_{2}^{2}}{2\left(\varphi_{3}-\varphi_{2}\right)}\right) I_{1} \\
& +\frac{1}{\alpha^{3}}\left[\left(\varphi(0)+C_{1}-3 \varphi_{2}\right) I_{2}-\frac{3\left(\varphi_{3}-\varphi_{2}\right)}{2} I_{3}\right] .
\end{aligned}
$$

Hence, from (25) and (32), we conclude

$$
\left\langle L^{-1} \varphi, \varphi\right\rangle=\frac{6 \beta^{2}}{T} \frac{\left(2-\kappa^{2}\right) K(\kappa)-3 E(\kappa)}{K^{2}(\kappa)} I(\kappa),
$$

where

$$
\begin{aligned}
& I(\kappa)=\frac{4\left(1-\kappa^{2}\right) K(\kappa)\left[E(\kappa)-\left(1-\kappa^{2}\right) K(\kappa)\right]}{\kappa^{2} \int_{0}^{K(\kappa)} \frac{\left(1-2 \kappa^{2}\right)-\left(2-\kappa^{2}\right) s n^{2}(x, \kappa)}{d n^{2}(x, \kappa)} d x} I_{1}^{2} \\
& +4\left[E(\kappa) K(\kappa)-\left(1-\kappa^{2}\right) K^{2}(\kappa)-\frac{\left(1-\kappa^{2}\right) K^{2}(\kappa)}{\int_{0}^{K(\kappa)} \frac{\left(1-2 \kappa^{2}\right)-\left(2-\kappa^{2}\right) s n^{2}(x, \kappa)}{d n^{2}(x, \kappa)} d x} I_{1}\right] I_{2}-3 \kappa^{2} K^{2}(\kappa) I_{3} .
\end{aligned}
$$

Note that the expression in front of $I(\kappa)$ in (33) is (positively) proportional to the speed $c$, which is positive in our case (i.e., $\kappa \in(0.98,1)$ ), and thus we need to show that $I(\kappa)<0, \kappa \in(0.98,1)$. Indeed this is the case, as the graph of the function $I(\kappa)$ shows; see Figure 2.

Thus, we find that $\lim _{\epsilon \rightarrow 0}\left\langle\mathcal{L}_{\epsilon}^{-1} \varphi_{\epsilon}, \varphi_{\epsilon}\right\rangle<0$ and hence $n\left(\left\langle\mathcal{L}_{\epsilon}^{-1} \varphi_{\epsilon},\right)\right\rangle=1$ for all small enough $\epsilon$. Consequently, $n\left(-H \mathcal{K}_{\epsilon}\right)=0$, which is to say that the waves are stable. 


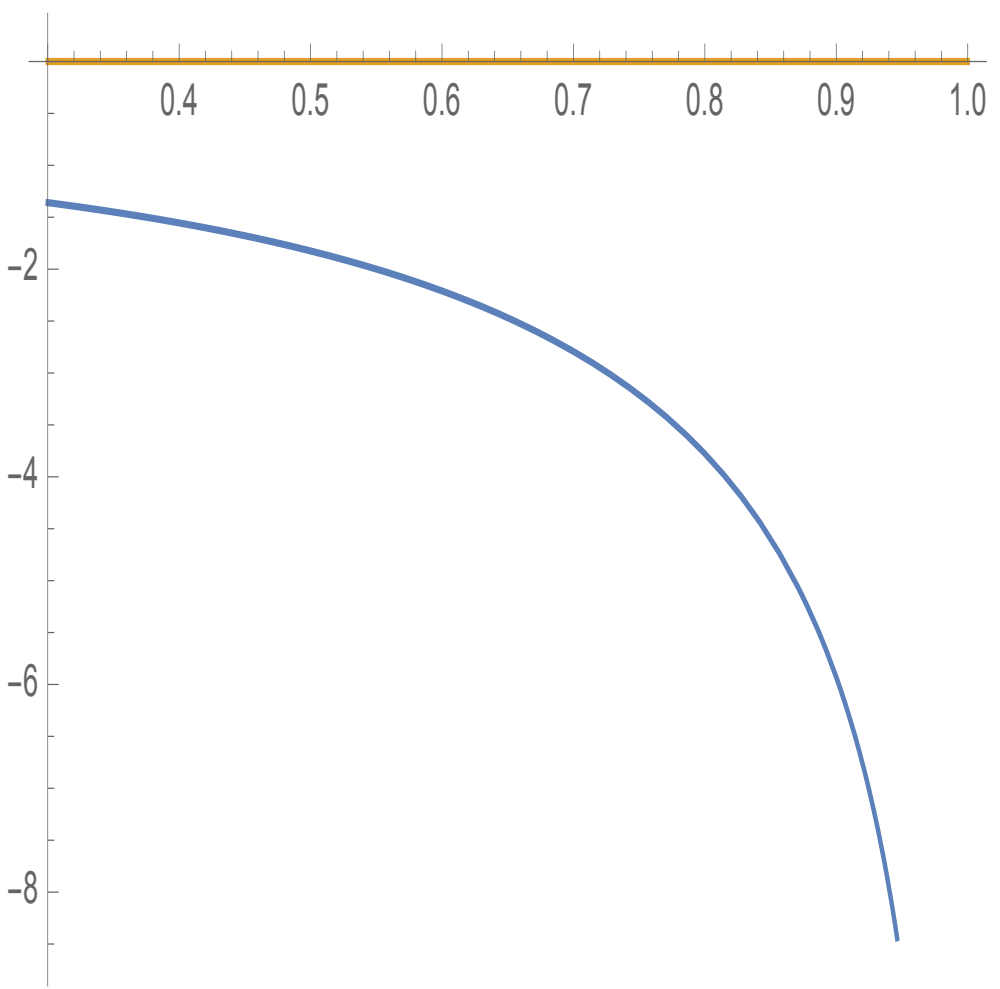

FIG. 2. The graph of the function $I(\kappa)$. Note that $I(\kappa)<0, \kappa \in(0.98,1)$.

4. Periodic traveling waves for the short-pulse equation. The generalized Ostrovsky equation that we consider is in the form (4), with $f(u)=-u^{p}$. For the purposes of this section, we consider the case $p=2$ only, although some of the other cases are certainly physically relevant and mathematically tractable.

For the profile equation, we impose the ansatz $u(x, t)=\varphi_{c}(x+c t), c>0$. We get

$$
c \varphi^{\prime \prime}=\varphi+\left(\varphi^{2}\right)_{x x}
$$

or

$$
\left[\varphi^{\prime}(c-2 \varphi)\right]^{\prime}=\varphi .
$$

At this point, we need to perform a well-known change of variables; see, for example, [10], although this originated much earlier (see [2, 24, 27, 31, 33, 34]). More specifically, take

$$
\xi=\eta-\frac{2 \Psi(\eta)}{c}=: \Xi(\eta),
$$

where $\varphi(\xi)=\Phi(\eta)=\Psi^{\prime}(\eta)$. Then (36) in the new variables takes the form

$$
c^{2} \Phi_{\eta \eta}=\Phi(c-2 \Phi) .
$$

Integrating once (37), we get

$$
\Phi_{\eta}^{2}=\frac{1}{c^{2}}\left[-\frac{4}{3} \Phi^{3}+c \Phi^{2}+A\right]=F(\Phi),
$$

Copyright (C) by SIAM. Unauthorized reproduction of this article is prohibited. 
where $A$ is a constant of integration. Clearly, $\Phi=\frac{c}{2}$ is a stationary solution of (36). This turns out to be a center. We are interested in constructing solutions that are close to this center. We have the following proposition.

Proposition 1. There exists $a_{0}>0$, so that for every $a:|a|<a_{0}$, there exists an even smooth function $\Phi_{a}$, solving (38), which is in the form

$$
\begin{aligned}
\Phi_{a}(\eta) & =\frac{c}{2}+a \cos \left(k_{a} \eta\right)+O\left(a^{2}\right):-\frac{2 \pi}{k_{a}} \leq \eta \leq \frac{2 \pi}{k_{a}}, \\
k_{a} & =\frac{1}{c}-\frac{10 a^{2}}{3 c^{2}}+O\left(a^{4}\right) .
\end{aligned}
$$

Proof. In the phase plane $\left(\Phi, \Phi^{\prime}\right)$ for $c>0,(37)$ has equilibria at $(0,0)$, which is saddle point, and at $\left(\frac{c}{2}, 0\right)$, which is a center. It is classical that the center is surrounded by a continuous band of periodic trajectories, which terminates at a separatrix, which is homoclinic to the saddle point. Introduce a small parameter $0<|a|<<1$. More precisely, for values ${ }^{10}$ of $A \in\left(-\frac{c^{3}}{12}, 0\right)$, the cubic polynomial $F_{A}(z)=-\frac{4}{3} z^{3}+c z^{2}+A$ has three real roots - one negative and two positive roots $\Phi_{0}, \Phi_{1}: 0<\Phi_{0}<\frac{c}{2}<\Phi_{1}$, and $\Phi_{0}<\Phi(x)<\Phi_{1}$. Then the period $T$ of $\Phi$ is given by

$$
T=\int_{0}^{T} d t=2 \int_{\Phi_{0}}^{\Phi_{1}} \frac{1}{F(X)} d X
$$

It is easy to see that the period $T$ is continuous function of $\Phi_{0}$, indeed by changing of variable

$$
X=\frac{\Phi_{1}-\Phi_{0}}{2} s+\frac{\Phi_{1}+\Phi_{0}}{2},
$$

and for $\Phi_{0} \rightarrow \frac{c}{2}$, we get

$$
T_{\Phi} \rightarrow T_{0}=2 \pi \sqrt{c}
$$

Let $\Phi(x)=\phi\left(k_{a} x\right)=\phi(z)$, where $\phi(z)$ is periodic function with period $2 \pi$. To conform with our earlier setup, we will take the interval in the form $[-\pi, \pi]$. We have

$$
k_{0}=\frac{2 \pi}{T_{0}}=\frac{1}{\sqrt{c}} .
$$

Then the function $\phi$ is given by the equation

$$
c^{2} k_{a}^{2} \phi^{\prime \prime}=c \phi-2 \phi^{2} .
$$

Let

$$
\begin{aligned}
\phi(z) & =\frac{c}{2}+a \cos (z)+a^{2} \phi_{2}(z)+a^{3} \phi_{3}(z)+O\left(a^{4}\right), \\
k_{a}^{2} & =\frac{1}{c}+a^{2} k_{1}+O\left(a^{4}\right) .
\end{aligned}
$$

We look for solutions parametrized by a small parameter $a$. Plugging in (42) into (41), we see that the coefficients of $O(a)$ are equal. For coefficients of $O\left(a^{2}\right)$, the compatibility condition is

$$
c\left(\phi_{2}^{\prime \prime}+\phi_{2}\right)=-2 \cos ^{2}(z)
$$

\footnotetext{
${ }^{10}$ When $a=0$, we obtain the center, $\Phi=\frac{c}{2}$ and $A=-\frac{c^{3}}{12}$.
} 
with solution $\phi_{2}(z)=\frac{1}{3 c}(\cos (2 z)-3)$. For $O\left(a^{3}\right)$, we have

$$
c\left(\phi_{3}^{\prime \prime}+\phi_{3}\right)=c^{2} k_{1} \cos (z)-4 \cos (z) \phi_{2}(z),
$$

which leads to the equality $k_{1}=-\frac{10}{3 c^{2}}$. Thus finally we get

$$
\phi(z)=\frac{c}{2}+a \cos (z)+a^{2} \frac{1}{3 c}(\cos (2 z)-3)+a^{3} \phi_{3}(z)+O\left(a^{4}\right),
$$

and

$$
k_{a}^{2}=\frac{1}{c}-a^{2} \frac{10}{3 c^{2}}+O\left(a^{4}\right) .
$$

Now, to construct the solutions to (35), we need to use the function $\Phi$ described in Proposition 1. In addition, we need to make sure that we restrict ourselves to an appropriate interval, where the transformation (36) is invertible. To that end, take the derivative $\Xi^{\prime}(\eta)$. We have

$$
\frac{d \xi}{d \eta}=\Xi^{\prime}(\eta)=1-\frac{2 \Psi^{\prime}(\eta)}{c}=1-\frac{2 \Phi(\eta)}{c} .
$$

From (42), we deduce that

$$
\frac{d \xi}{d \eta}=\Xi^{\prime}(\eta)=1-\frac{2}{c}\left(\frac{c}{2}+a \cos \left(k_{a} \eta\right)+O\left(a^{2}\right)\right)=-\frac{2 a}{c} \cos \left(k_{a} \eta\right)+O\left(a^{2}\right) .
$$

Thus, we need to restrict $\eta$ to an interval, where the $\cos \left(k_{a} \eta\right)$ does not vanish, for example,

$$
-\frac{B}{k_{a}} \leq \eta \leq \frac{B}{k_{a}} .
$$

where $0<B<\frac{\pi}{2}$. Now that we know that $\Xi(\eta):\left[-B / k_{a}, B / k_{a}\right] \rightarrow \mathbf{R}^{1}$ has an inverse function, say, $\eta(\xi)$, we need to determine the domain of the inverse function. Observing that

$$
\begin{aligned}
& \Xi^{\prime}(\eta)=-\frac{2 a}{c} \cos \left(k_{a} \eta\right)+O\left(a^{2}\right)<0, \text { when } a>0, \\
& \Xi^{\prime}(\eta)=-\frac{2 a}{c} \cos \left(k_{a} \eta\right)+O\left(a^{2}\right)>0, \text { when } a<0 .
\end{aligned}
$$

We define the positive quantity $\xi_{a}$ as follows:

$$
\begin{aligned}
-\xi_{a} & =\Xi\left(\frac{B}{k_{a}}\right) \text { when } a>0, \\
\xi_{a} & =\Xi\left(\frac{B}{k_{a}}\right) \text { when } a<0 .
\end{aligned}
$$

With this in mind, we just take

$$
\varphi(\xi):=\phi\left(k_{a} \eta(\xi)\right), \varphi:\left[-\xi_{a}, \xi_{a}\right] \rightarrow \mathbf{R}^{1},
$$

where $\eta(\xi)$ is the inverse function to the one defined in (36).

By construction, $\varphi$ solves (35). It is also obviously an even function. We now verify the periodicity on the interval $\left[-\xi_{a}, \xi_{a}\right]$. We have

$$
\varphi\left(\xi_{a}\right)=\phi\left(k_{a} \eta\left(\xi_{a}\right)\right)=\phi(-B), \varphi\left(-\xi_{a}\right)=\phi\left(k_{a} \eta\left(-\xi_{a}\right)\right)=\phi(B) .
$$

Thus, $\varphi\left(\xi_{a}\right)=\varphi\left(-\xi_{a}\right)$, since $\phi$ is an even function.

Copyright $@$ by SIAM. Unauthorized reproduction of this article is prohibited. 
Furthermore,

$$
\varphi^{\prime}(\xi)=k_{a} \phi^{\prime}\left(k_{a} \eta(\xi)\right) \eta^{\prime}(\xi)=k_{a} \frac{\phi^{\prime}\left(k_{a} \eta(\xi)\right)}{\frac{d \xi}{d \eta}}=k_{a} \frac{\phi^{\prime}\left(k_{a} \eta(\xi)\right)}{1-\frac{2}{c} \varphi(\xi)}
$$

Evaluating at $\xi= \pm \xi_{a}$, we have that

$$
1-\frac{2}{c} \varphi\left( \pm \xi_{a}\right)=1-\frac{2}{c} \phi(\mp B)=1-\frac{2}{c}\left(\frac{c}{2}+a \cos (\mp B)+O\left(a^{2}\right)\right)=-\frac{2 a}{c} \cos (B)+O\left(a^{2}\right) \neq 0,
$$

whence the denominator is nonzero for small enough $a$. Also, for the numerator, we have

$$
\phi^{\prime}\left(k_{a} \eta\left(\xi_{a}\right)\right)=\phi^{\prime}(-B)=-\phi^{\prime}(B)=-\phi^{\prime}\left(k_{a} \eta\left(-\xi_{a}\right)\right),
$$

since $\phi^{\prime}$ is an odd function. We conclude that

$$
\varphi^{\prime}\left(-\xi_{a}\right)=-\varphi^{\prime}\left(\xi_{a}\right)
$$

That is, the function has a corner crest at the endpoints of the interval $\pm \xi_{a}$. If one takes a periodization of such function, the corner crests will of course appear at all points in the form $(2 k+1) \xi_{a}, k \in \mathcal{Z}$. This is the peakon type solution that we have discussed, see Figure 3 below.

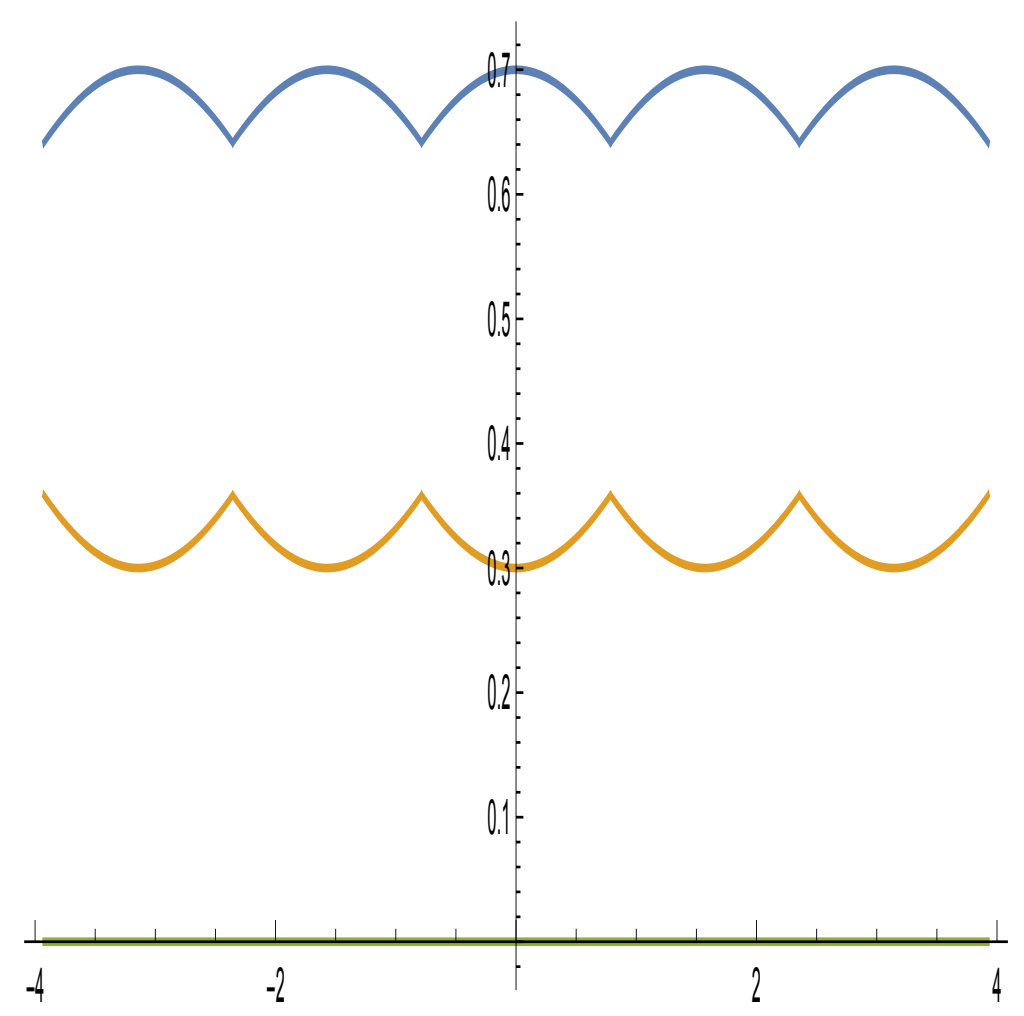

FIG. 3. Peakons: the blue one is with $a>0$, the yellow one is with $a<0$. 
THEOREM 3 (Existence of peakons solutions). There exists $a_{0}>0$, so that for each $a:|a|<a_{0}$, there is a one-parameter family of even functions $\varphi_{a, b}, \varphi_{a, B}$ : $\left[-\xi_{a}, \xi_{a}\right] \rightarrow \mathbf{R}^{1}, B \in(0, \pi / 2)$, which classically solve (35) in the interval $\left(-\xi_{a}, \xi_{a}\right)$. These functions are $C^{\infty}\left(-\xi_{a}, \xi_{a}\right)$.

Moreover, the $2 \xi_{\text {a }}$ periodizations of these functions remain continuous functions, but their derivatives develop jump discontinuities at the points $(2 k+1) \xi_{a}, k \in \mathcal{Z}$.

5. Spectral stability of the traveling peakons for the short pulse equation. In this section, we show the spectral stability for the peakons constructed in section 4 . The change of variables (36), which "translates" the peakon equation (35) into the more tractable Schrödinger equation (36) will play pivotal role in the problem for linear/spectral stability as well.

More concretely, suppose that $\varphi_{c}$ is a solution ${ }^{11}$ of $(35)$ in the interval $[-T, T]$, where we henceforth set $T:=\xi_{a}$. Introduce the ansatz $u(t, x)=\varphi_{c}(x+c t)+v(t, x+c t)$ in (34). Ignoring quadratic and higher order terms, and adopting the new variable $x+c t \rightarrow \xi$, we arrive at

$$
\left(v_{t}+((c-2 \varphi) v)_{\xi}\right)_{\xi}=v, \quad-T \leq \xi \leq T,
$$

where $v$ is a periodic function in $[-T, T]$. We consider the stability problem for (46). Take $v(t, \xi)=e^{\lambda t} w(\xi)$, with the requirement that ${ }^{12} w \in H^{2}[-T, T]$. The problem (46) turns into the eigenvalue problem

$$
\left(\lambda w+((c-2 \varphi) w)_{\xi}\right)_{\xi}=w, \quad-T \leq \xi \leq T .
$$

Equivalently, we ask that the $2 T$ periodization of $w$ and $\varphi$ (also denoted by $w, \varphi$ ) obeys

$$
\left(\lambda w+((c-2 \varphi) w)_{\xi}\right)_{\xi}=w, \xi \in \mathbf{R}^{1} .
$$

In particular, it must be that the function $\xi \rightarrow \lambda w+((c-2 \varphi) w)_{\xi}$ is continuous at $\pm T$. By the continuity of $w, w_{\xi}, \varphi$ at $\pm T$ and the fact that $\varphi^{\prime}(-T+)=-\varphi^{\prime}(T-) \neq 0$, we conclude that $w( \pm T)=0$. Note that by the periodicity of $w_{\xi}$ and $w(-T)=w(T)=0$

$$
\int_{-T}^{T} w(\xi) d \xi=\left.\left[\lambda w+((c-2 \varphi) w)_{\xi}\right]\right|_{-T} ^{T}=0
$$

Thus, one can select a function $z: \int_{-T}^{T} z(\xi)=0$, so that $w=z_{\xi}$. Since we require that $w \in C^{2}[-T, T]$ and periodic, we have that $w \in H^{2}[-T, T]$, whence $z \in H^{3}[-T, T]$.

We have

$$
\left(\lambda z_{\xi}+\left((c-2 \varphi) z_{\xi}\right)_{\xi}\right)_{\xi}=z_{\xi}, \quad-T<\xi<T .
$$

Integrating once in $\xi$ allows us to conclude that

$$
\lambda z_{\xi}+\left((c-2 \varphi) z_{\xi}\right)_{\xi}=z+C, \quad-T<\xi<T,
$$

for some constant of integration $C$. Since $\int_{-T}^{T} z(\xi)=0, C=0$ and we obtain

$$
\lambda z_{\xi}+\left((c-2 \varphi) z_{\xi}\right)_{\xi}=z, \quad-T<\xi<T .
$$

\footnotetext{
${ }^{11}$ In the sense of Theorem 3.

${ }^{12}$ In particular, the periodization of $w, w_{\xi}$ are continuous everywhere.
} 
By our construction of the peakons, the interval $[-T, T]$ is such that the transformation $\xi=\Xi(\eta)$ is a one-to-one mapping from $\left[-B / k_{a}, B / k_{a}\right]$ onto $[-T, T]$. Introduce $b:=\frac{B}{k_{a}}$. Take $\eta(\xi):[-T, T] \rightarrow[-b, b]$ to be the inverse of $\Xi(\eta)$. Thus, we may introduce a new function $Z$, via

$$
z(\xi)=Z(\eta(\xi))
$$

Note that while $z$ enjoys the mean value zero property, this is clearly no longer the case for $Z$. We now compute the derivatives of $z$ in terms of $Z$. We have

$$
z_{\xi}(\xi)=\frac{Z_{\eta}}{\frac{d \xi}{d \eta}}=\frac{Z_{\eta}}{1-\frac{2}{c} \varphi(\xi)}=\frac{c Z_{\eta}}{c-2 \varphi(\xi)}
$$

Thus, $(c-2 \varphi) z_{\xi}=c Z_{\eta}$, and hence

$$
\left[(c-2 \varphi) z_{\xi}\right]_{\xi}=c \frac{d}{d \xi}\left(Z_{\eta}(\eta(\xi))\right)=\frac{c Z_{\eta \eta}}{\frac{d \xi}{d \eta}}=\frac{c^{2} Z_{\eta \eta}}{c-2 \varphi(\xi)} .
$$

Plugging (51), (52) into (49) and taking into account that $\varphi(\xi)=\Phi(\eta)$ leads us to the new spectral problem

$$
-c^{2} Z_{\eta \eta}+c Z-2 \Phi Z=\lambda c Z_{\eta},-b<\eta<b .
$$

Let us now show that the function $Z$ in $(50)$ is in fact $Z \in H^{2}[-b, b]$. First of all, the formula (50) ensures that $Z$ is continuous and $2 b$ periodic. Next, the relation $Z_{\eta}=c^{-1}(c-2 \varphi) z_{\xi}$ implies that $Z_{\eta}$ is continuous and $2 b$ periodic. Finally, (53) implies that $Z_{\eta \eta}$ is a linear combination of continuous functions on $[-b, b]$ and hence $Z \in H^{2}[-b, b]$. Thus, we have shown that if the eigenvalue problem (48) has a solution $w \in H^{2}$, then the transformed problem (53) will have a solution $Z \in H^{2}[-b, b]$. For the rest of the section, we show that this is not the case, hence we will conclude stability for the peakon solutions.

Since we are looking for solutions of (53) with $\Re \lambda>0$ and $c>0$, we can rewrite the spectral problem in the form

$$
\mathcal{L}[Z]=\mu Z^{\prime}
$$

where $\mu=\lambda c$, and

$$
\mathcal{L}=\mathcal{L}_{a}:=-c^{2} \partial_{y y}+c-2 \Phi,-b \leq y \leq b,
$$

where $Z \in D(\mathcal{L})=H^{2}[-b, b]$. Here, recall that the function $\Phi$ was constructed explicitly in Proposition 1. Clearly, the instability occurs for (53), exactly when it occurs for (54), because $\Re(\mu)=c \Re(\lambda)$ and hence $\Re(\mu)$ and $\Re(\lambda)$ have the same sign. In other words, we study (54) for stability/instability.

Let $P_{0}: L^{2}[-b, b] \rightarrow L_{0}^{2}[-b, b]$ be the projection onto $L_{0}^{2}$

$$
P_{0} f(y)=f(y)-\frac{1}{2 b} \int_{-b}^{b} f(z) d z .
$$

Introduce the operator $\mathcal{L}_{a}^{0}:=P_{0} \mathcal{L}_{a} P_{0}$, which is self-adjoint, when equipped with the domain $D\left(\mathcal{L}_{a}^{0}\right)=H_{0}^{2}[-b, b]$. Alternatively, one may define it via the quadratic form $q(u, v)=\langle\mathcal{L} u, v\rangle$, where $u, v \in H_{0}^{1}[-b, b]$. We will need the following lemma regarding the spectrum of $\mathcal{L}$. 
Lemma 2. The spectrum of $\mathcal{L}_{a}$ consists of eigenvalues only, each with finite multiplicity. There exists $a_{0}>0$ so that for all $-a_{0}<a<0$, the operator $\mathcal{L}_{a}$ is a positive operator. For all $a: 0<a<a_{0}, \mathcal{L}_{a}$ has one negative eigenvalue, denoted by $\lambda_{0}(\mathcal{L})$, for which $\frac{\left|\lambda_{0}\left(\mathcal{L}_{a}\right)\right|}{a} \sim 1$ and the rest of the spectrum is positive. In particular, $\mathcal{L}_{a}$ is invertible for all $a:|a|<a_{0}$. Finally, there exists $\delta=\delta_{b}$ so that $\mathcal{L}_{a}^{0} \geq \delta I d$.

Proof. Clearly, the spectrum of $\mathcal{L}_{a}$ consists entirely of real eigenvalues, each with finite multiplicity. This is because $\mathcal{L}$ is bounded from below and also $\mathcal{L}$ can be represented as a sum of $A=-\partial_{y y}$ plus a relative compact perturbation. We have by (43)

$$
\begin{aligned}
\mathcal{L}_{a} & =-c^{2} \partial_{y y}+c-2 \Phi=-c^{2} \partial_{y y}+c-2\left(\frac{c}{2}+a \cos \left(k_{a} y\right)+O\left(a^{2}\right)\right) \\
& =-c^{2} \partial_{y y}-2 a \cos \left(k_{a} y\right)+O\left(a^{2}\right) .
\end{aligned}
$$

Recall that $y \in(-b, b)$ in which $\cos \left(k_{a} y\right)>0$. In fact, $\cos \left(k_{a} y\right) \geq \sigma_{b}>0$, whence

$$
\mathcal{L}_{a} \geq-c^{2} \partial_{y y}-2 a \sigma_{b} \geq-2 a \sigma_{b}=2|a| \sigma_{b} .
$$

Thus, we immediately conclude that for all $a<0,|a|<<1$, we have that $\mathcal{L}$ is a positive operator.

For $a>0$, we observe that

$$
\mathcal{L}_{a}=-c^{2} \partial_{y y}-2 a \cos \left(k_{a} y\right)+O\left(a^{2}\right) \geq-2 a \cos \left(k_{a} y\right)+O\left(a^{2}\right) \geq-2 a,
$$

whence $\lambda_{0}(\mathcal{L}) \geq-2 a$. On the other hand, using the expression for $\Phi$ from Proposition 1

$$
\left\langle\mathcal{L}_{a}(1), 1\right\rangle=\langle c-2 \Phi, 1\rangle=-2 a\left\langle 1, \cos \left(k_{a} \cdot\right)\right\rangle+O\left(a^{2}\right) \leq-a b \sigma_{b}
$$

for $a:|a|<<1$, since $\cos \left(k_{a} y\right) \geq \sigma_{b}>0, y \in(-b, b)$.

Finally, restrict to the mean value zero subspace $L_{0}^{2}[-b, b]$, which is co-dimension one subspace. We have for $f \in H_{0}^{1}[-b, b]$

$$
\left\langle\mathcal{L}_{a} f, f\right\rangle=c^{2}\left\|f^{\prime}\right\|^{2}-2 a \int_{-b}^{b} f^{2}(y) \cos \left(k_{a} y\right) d y+O\left(a^{2}\right) .
$$

On the other hand, for $f \in H_{0}^{1}[-b, b]$, there is the Poincaré inequality, $\left\|f^{\prime}\right\| \geq \frac{\pi}{b}\|f\|$. Thus, we conclude that for all $a:|a|<<1$ and for all for $f \in H_{0}^{1}[-b, b]$

$$
\left\langle\mathcal{L}_{a} f, f\right\rangle \geq\left(\frac{c^{2} \pi^{2}}{b^{2}}-2 a+O\left(a^{2}\right)\right)\|f\|^{2} \geq \frac{c^{2} \pi^{2}}{2 b^{2}}\|f\|^{2} .
$$

Note that this last inequality incidentally establishes the last claim in Lemma 2.

By the min-max characterization of the eigenvalues, the last inequality implies that $\lambda_{1}(\mathcal{L})$ is positive, bounded away from zero (in terms of $a$ ). Combining the last observations implies that for $0<a<<1, \mathcal{L}_{a}$ has a small (and simple) negative eigenvalue and the rest of the spectrum is contained in $\left(k_{b}, \infty\right)$ for some $k>0$.

In addition, we can deduce that in fact the negative eigenvalue $\left|\lambda_{0}\left(\mathcal{L}_{a}\right)\right| \sim a$. More precisely, there exist $0<c_{0} \leq 2$ so that for all $0<a<<1$,

$$
c_{0} \leq \frac{\left|\lambda_{0}\left(\mathcal{L}_{a}\right)\right|}{a} \leq 2
$$

Indeed, the right-hand-side inequality follows from our earlier estimate $\lambda_{0}\left(\mathcal{L}_{a}\right) \geq-2 a$, while the left-hand-side inequality eventually follows from (55). 
To that end, let $\psi_{0},\left\{\psi_{j}\right\}_{j}$ be an enumeration of the eigenfunctions of $\mathcal{L}_{a}$, corresponding to eigenvalues $\lambda_{0}\left(\mathcal{L}_{a}\right)<0,0<c_{b} \leq \lambda_{1}\left(\mathcal{L}_{a}\right) \leq \lambda_{2}\left(\mathcal{L}_{a}\right) \ldots$ We have

$$
-2 a b \sigma_{b} \geq\left\langle\mathcal{L}_{a}(1), 1\right\rangle=\lambda_{0}\left(\mathcal{L}_{a}\right)\left\langle 1, \psi_{0}\right\rangle^{2}+\sum_{j=1}^{\infty} \lambda_{j}\left(\mathcal{L}_{a}\right)\left\langle 1, \psi_{j}\right\rangle^{2} \geq \lambda_{0}\left(\mathcal{L}_{a}\right)\left\langle 1, \psi_{0}\right\rangle^{2} .
$$

Noting that $\left|\left\langle 1, \psi_{0}\right\rangle\right| \leq\|1\|\left\|\psi_{0}\right\|=C$, we conclude that $-2 a b \sigma_{b} \geq-C\left|\lambda_{0}\left(\mathcal{L}_{a}\right)\right|$. But this last inequality means $\left|\lambda_{0}(\mathcal{L})\right| \geq c_{0} a$, whence (56) is established in full.

Having the results of Lemma 2 , we return to the consideration of the eigenvalue problem (54). Introduce a new variable $W: W=\mathcal{L}[Z]$ or $Z=\mathcal{L}^{-1}[W]$, since $\mathcal{L}$ is invertible. This allows us to rewrite (54) as

$$
W=\mu \partial_{x}\left[\mathcal{L}^{-1} W\right] .
$$

Here, it is worth noting that the theory developed in [9] applies to the stability of (57). We provide the short proof below using only elementary arguments.

Since the right-hand side of the last equation is an exact derivative, $W$ has mean value zero or $W \in L_{0}^{2}[-b, b]$. In fact, keeping track of the derivatives ${ }^{13}$ allows us to conclude $W \in H_{0}^{1}[-b, b]$. Thus, we introduce yet another new variable $Q \in H_{0}^{3 / 2}[-b, b]$, with $W=\left|\partial_{x}\right|^{1 / 2} Q$. Taking into account $\partial_{x}=-H\left|\partial_{x}\right|$, we can rewrite (57) as follows:

$$
-H\left|\partial_{x}\right|^{1 / 2} \mathcal{L}^{-1}\left|\partial_{x}\right|^{1 / 2} Q=\mu^{-1} Q .
$$

Observe that in (58) $\mathcal{L}^{-1}$ acts upon $\left|\partial_{x}\right|^{1 / 2} Q \in L_{0}^{2}[-b, b]$ and then the operator $\left|\partial_{x}\right|^{1 / 2}$ in front of $\mathcal{L}^{-1}$ can be factorized $\left|\partial_{x}\right|^{1 / 2}=\left|\partial_{x}\right|^{1 / 2} P_{0}$. Thus, we may rewrite (58)

$$
-H\left|\partial_{x}\right|^{1 / 2}\left[\mathcal{L}^{0}\right]^{-1}\left|\partial_{x}\right|^{1 / 2} Q=\mu^{-1} Q
$$

Note that in this particular form, the eigenvalue problem fits the framework of $[15,16]$. Indeed, with $J:=-H$ and $L:=\left|\partial_{x}\right|^{1 / 2}\left[\mathcal{L}^{0}\right]^{-1}\left|\partial_{x}\right|^{1 / 2}$, we have a pair of anti-self-adjoint and self-adjoint operators, so that the eigenvalue problem of interest is in the form $J L Q=\mu^{-1} Q$. Moreover, we have that $L>0$, since for every $f \in H_{0}^{2}[-b, b]$

$$
\langle L f, f\rangle=\left\langle\left[\mathcal{L}^{0}\right]^{-1}\left|\partial_{x}\right|^{1 / 2} f,\left|\partial_{x}\right|^{1 / 2} f\right\rangle>0,
$$

since $\mathcal{L}^{0}>\delta I d$ by virtue of Lemma 2 . Thus, $L>0$. Based on that, we can now conclude that the eigenvalue problem $J L Q=h Q$ cannot have solutions with $\Re h>0$, which implies that (58) and subsequently (54) do not have unstable solutions. This follows by a simple application of the general results in $[15,16]$ (and maybe as a consequence of some older papers). Let us, however, provide a simple alternative proof.

Assume that $J L Q=h Q$ has unstable solutions, that is,

$$
J L\left(Q_{1}+i Q_{2}\right)=\left(h_{1}+i h_{2}\right)\left(Q_{1}+i Q_{2}\right),
$$

with $h_{1}>0$ and $\left(Q_{1}, Q_{2}\right) \neq(0,0)$. Taking real and imaginary parts yields the system

$$
\mid \begin{aligned}
& J L Q_{1}=h_{1} Q_{1}-h_{2} Q_{2} \\
& J L Q_{2}=h_{2} Q_{1}+h_{1} Q_{2}
\end{aligned}
$$

\footnotetext{
${ }^{13}$ By definition $\mathcal{L}^{-1}[W] \in H^{2}[-b, b]$, whence $\partial_{x} \mathcal{L}^{-1}[W] \in H^{1}[-b, b]$.
} 
Taking dot products with $L Q_{1}, L Q_{2}$, respectively, and taking into account $\langle J f, f\rangle=0$ for all real-valued functions, ${ }^{14}$ we obtain

$$
\mid \begin{aligned}
& h_{1}\left\langle Q_{1}, L Q_{1}\right\rangle-h_{2}\left\langle Q_{2}, L Q_{1}\right\rangle=0 \\
& h_{2}\left\langle Q_{1}, L Q_{2}\right\rangle+h_{1}\left\langle Q_{2}, L Q_{2}\right\rangle=0
\end{aligned}
$$

By the self-adjointness of $L$ and since $Q_{1}, Q_{2}$ are real, $\left\langle Q_{2}, L Q_{1}\right\rangle=\left\langle Q_{1}, L Q_{2}\right\rangle$. Thus, adding the equations results in

$$
h_{1}\left(\left\langle Q_{1}, L Q_{1}\right\rangle+\left\langle Q_{2}, L Q_{2}\right\rangle\right)=0 .
$$

Recall though that $L>0$, whence $\left(\left\langle Q_{1}, L Q_{1}\right\rangle+\left\langle Q_{2}, L Q_{2}\right\rangle>\delta\left(\left\|Q_{1}\right\|^{2}+\left\|Q_{2}\right\|^{2}\right)\right.$. Then, either $Q_{1}=Q_{2}=0$ or $h_{1}=0$, a contradiction.

We have thus proved the main result.

THEOREM 4. There exists $a_{0}>0$ so that for all $a:|a|<a_{0}$, the waves $\varphi_{a}$ constructed in section 4 are spectrally stable.

\section{REFERENCES}

[1] J. Angulo, J. Bona, And M. Scialom, Stability of cnoidal waves, Adv. Diferential Equations, 11 (2006), pp. 1321-1374.

[2] J. P. BoYD, Ostrovsky and Hunter's generic wave equation for weakly dispersive waves: Matched asymptotic and pseudo-spectral study of the paraboloidal travelling waves (corner and near-corner waves), European J. Appl. Math., 16 (2004), pp. 65-81.

[3] J. Bronski and M. Johnson, The modulational instability for a generalized KdV equation, Arch. Ration. Mech. Anal., 2 (2010), pp. 357-400.

[4] J. Bronski, M. Johnson, And T. Kapitula, An index theorem for the stability of periodic travelling waves of KdV type, Proc. Roy. Soc. Edinburgh Sect. A, 6 (2011), pp. 1141-1173.

[5] V. Bruneau and E. M. Ouhabaz, Lieb-Thirring estimates for non-self-adjoint Schrödinger operators, J. Math. Phys., 49 (2008).

[6] M. Chugunova And D. Pelinovsky, Count of eigenvalues in the generalized eigenvalue problem, J. Math. Phys., 51 (2010), 052901.

[7] N. Costanzino, V. Manukian, and C. K. R. T. Jones, Solitary waves of the regularized short pulse and Ostrovsky equations, SIAM J. Math. Anal., 41 (2009), pp. 2088-2106.

[8] N. Costanzino, V. Manukian, C. K. R. T. Jones, and B. Sandstede, Existence of multipulses of the regularized short-pulse and Ostrovsky equations, J. Dynam. Differential Equations, 21 (2009), pp. 607-622.

[9] B. DeCONINCK AND T. KAPITUla, On the spectral and orbital stability of spetially periodic stationary solutions of generalized Koerteweg-de Vries equations, Hamiltonian Partial Differential Equations Appl., 75 (2015), pp. 285-322.

[10] R. Grimshaw, K. Helfrich, and E. R. Johnson, The reduced Ostrovsky equation: Integrability and breaking, Stud. Appl. Math., 129 (2012), pp. 414-436.

[11] R. Grimshaw and D. Pelinovsky, Global existence of small-norm solutions in the reduced Ostrovsky equation, Discrete Contin. Dyn. Syst., 34 (2014), pp. 557-566.

[12] S. Hakkaev, I. Iliev, AND K. Kirchev, Stability of periodic travelling shallow-water waves determined by Newton's equation, J. Phys. A, 41 (2008), 085203.

[13] J. K. Hunter, Numerical solution of some nonlinear dispersive wave equations, in Computational Solution of Nonlinear Systems of Equations, Lectures in Appl. Math., 26, E. L. Allgower and K. Georg, eds., AMS, Providence, RI, 1990, pp. 301-316.

[14] D. Henry, Geometric Theory of Semilinear Parabolic Equations, Lecture Notes in Math. 840, Springer-Verlag, Berlin, 1981.

[15] T. Kapitula, P. G. Kevrekidis, and B. Sandstede, Counting eigenvalues via the Krein signature in infinite-dimensional Hamiltonian systems, Phys. D, 195 (2004), pp. 263-282.

[16] T. Kapitula, P. G. Kevrekidis, and B. Sandstede, Addendum: "Counting eigenvalues via the Krein signature in infinite-dimensional Hamiltonian systems" Phys. D 195 (2004), no. 3-4, 263-282, Phys. D, 201 (2005), pp. 199-201.

\footnotetext{
${ }^{14}$ This property is of course valid for all skew-symmetric operators $J$, which map real-valued into real-valued functions.
}

Copyright (c) by SIAM. Unauthorized reproduction of this article is prohibited. 
[17] T. Kapitula and K. Promislow, Spectral and Dynamical Stability of Nonlinear Waves, Appl. Math. Sci. 185, Springer, New York, 2013.

[18] S. Levandosky AND Y. Liu, Stability of solitary waves of a generalized Ostrovsky equation, SIAM J. Math. Anal., 38 (2006), pp. 985-1011.

[19] S. Levandosky And Y. Liu, Stability and weak rotation limit of solitary waves of the Ostrovsky equation, Discrete Contin. Dyn. Syst. Ser. B, 7 (2007), pp. 793-806.

[20] Y. Liu, On the stability of solitary waves for the Ostrovsky equation, Quart. Appl. Math., 65 (2007), pp. 571-589.

[21] Y. LiU And M. OHta, Stability of solitary waves for the Ostrovsky equation, Proc. Amer. Math. Soc., 136 (2008), pp. 511-517.

[22] Y. Liu, D. Pelinovsky, and A. Sakovich, Wave breaking in the short-pulse equation, Dyn. Partial Differential Equations, 6 (2009), pp. 291-310.

[23] Y. Liu, D. Pelinovsky, And A. Sakovich, Wave breaking in the Ostrovsky-Hunter equation, SIAM J. Math. Anal., 42 (2010), pp. 1967-1985.

[24] A. J. Morrisson, E. J. Parkes, and V. O. Vakhnenko, The $N$ loop soliton solutions of the Vakhnenko equation, Nonlinearity, 12 (1999), pp. 1427-1437.

[25] L. A. Ostrovsky, Nonlinear internal waves a in rotating ocean, Oceanology, 18 (1978), pp. 119-125.

[26] E. J. PARkes, The stability of solutions of Vakhnenko's equation, J. Phys. A, 26 (1993), pp. 6469-6475.

[27] E. K. PARKes, Explicit solutions of the reduced Ostrovsky equation, Chaos Solitons Fractals, 31 (2007), pp. 602-610.

[28] A. Sakovich and S. Sakovich, Solitary wave solutions of the short pulse equation, J. Phys. A, 39 (2006), pp. L361-L367.

[29] T. SchäFER AND C. E. WAYne, Propagation of ultra-short optical pulses in cubic nonlinear media, Phys. D, 196 (2004), pp. 90-105.

[30] A. Stefanov, Y. Shen, and P. Kevrekidis, Well-posedness and small data scattering for the generalized Ostrovsky equation, J. Differential Equations, 249 (2010), pp. 2600-2617.

[31] Y. A. Stepanyants, On stationary solutions of the reduced Ostrovsky equation: Periodic waves, compactons and compound solitons, Chaos Solitons Fractals, 28 (2006) pp. 193-204.

[32] V. A. VAkhnenko, Solitons in a nonlinear model medium, J. Phys. A, 25 (1992), pp. 4181-4187.

[33] V. O. Vakhnenko and E. J. Parkes, The two loop soliton solution of the Vakhnenko equation, Nonlinearity, 11 (1998), pp. 1457-1464.

[34] V. O. VAKhnenko And E. J. PARKes, The calculation of multi-soliton solutions of the Vakhnenko equation by the inverse scattering method, Chaos Solitons Fractals, 13 (2002), pp. 1819-1826.

Copyright (c) by SIAM. Unauthorized reproduction of this article is prohibited. 